\title{
FINITE ELEMENT APPROXIMATION OF SINGULAR POWER-LAW SYSTEMS
}

\begin{abstract}
ADRIAN HIRN
Abstract. Non-Newtonian fluid motions are often modeled by a power-law ansatz. In the present paper, we consider shear thinning singular power-law models which feature an unbounded viscosity in the limit of zero shear rate, and we study the finite element (FE) discretization of the equations of motion. In the case under consideration, numerical instabilities usually arise when the FE equations are solved via Newton's method. In this paper, we propose a numerical method that enables the stable approximation of singular powerlaw systems and that is based on a simple regularization of the power-law model. Our proposed method generates a sequence of discrete functions that is computable in practice via Newton's method and that converges to the exact solution of the power-law system for diminishing mesh size. First, for the regularized model we discuss Newton's method and we show its stability in the sense that we derive an upper bound for the condition number of the Newton matrix. Then, we prove a priori error estimates that quantify the convergence of the proposed method. Finally, we illustrate numerically that our regularized approximation method surpasses the nonregularized one regarding accuracy and numerical efficiency.
\end{abstract}

\section{INTRODUCTION}

We study the steady flow of an incompressible homogeneous fluid in a bounded domain $\Omega \subset \mathbb{R}^{d}, d \in\{2,3\}$, governed by the following system of PDEs:

$$
\left.\begin{array}{rl}
-\operatorname{div} \mathcal{S}(\boldsymbol{D} \boldsymbol{v})+\nabla \pi & =\boldsymbol{f} \\
\operatorname{div} \boldsymbol{v} & =0
\end{array}\right\} \quad \text { in } \Omega .
$$

Here, $\boldsymbol{v}=\left(v_{1}, \ldots, v_{d}\right)$ is the velocity, $\pi$ denotes the kinematic pressure, and $\boldsymbol{f}$ describes an external body force. The symbol $\boldsymbol{D} \boldsymbol{v}$ stands for the symmetric part of the velocity gradient $\nabla \boldsymbol{v}$, i.e., $\boldsymbol{D} \boldsymbol{v}:=\frac{1}{2}\left(\nabla \boldsymbol{v}+\nabla \boldsymbol{v}^{\top}\right)$. Note that we neglect inertial forces in (1.1) 1 and, hence, we avoid mathematical difficulties related to the convective term. In this paper we consider the extra stress tensors

$$
\mathcal{S}(\boldsymbol{D} \boldsymbol{v}):=\mu\left(|\boldsymbol{D} \boldsymbol{v}|^{2}\right) \boldsymbol{D} \boldsymbol{v}
$$

in which, for $p>1, \varepsilon \geq 0$, the generalized kinematic viscosity $\mu$ is given by

$$
\mu\left(|\boldsymbol{D} \boldsymbol{v}|^{2}\right):=\mu_{0}\left(\varepsilon^{2}+|\boldsymbol{D} \boldsymbol{v}|^{2}\right)^{\frac{p-2}{2}} .
$$

Received by the editor March 2, 2011 and, in revised form, November 4, 2011.

2010 Mathematics Subject Classification. Primary 76A05, 35Q35, 65N30, 65N12, 65N15.

Key words and phrases. Power-law fluid, finite element method, error analysis.

This work was supported by the International Graduate College IGK 710 "Complex Processes: Modeling, Simulation and Optimization" and the Heidelberg Graduate School of Mathematical and Computational Methods for the Sciences (HGS MathComp) at the Interdisciplinary Center for Scientific Computing (IWR) of the University of Heidelberg.

(c)2013 American Mathematical Society Reverts to public domain 28 years from publication 
For $\varepsilon=0$ the model (1.3) is referred to as the power-law model. For $p<2$ the power-law model features an unbounded viscosity in the limit of zero shear rate $(|\boldsymbol{D} \boldsymbol{v}| \searrow 0)$ and, hence, for $\varepsilon=0$ and $p<2$ the equations of motion (1.1)-(1.3) are called singular power-law systems. For $\varepsilon>0$ the model (1.3) represents the Carreau model which regularizes the singular power-law model. The model (1.3) is frequently used in engineering, blood rheology, and geology (see, e.g., 6, 21, 12]). Concerning models of type (1.3), extensive discussions can also be found in [19, 20. Most real fluids modeled by (1.3) exhibit shear thinning behavior that corresponds to exponents $p<2$. This paper is restricted to the shear thinning case.

We assume that the boundary $\partial \Omega$ of the domain $\Omega$ is Lipschitz. Then, we complement system (1.1) with homogeneous Dirichlet boundary conditions

$$
\boldsymbol{v}=\mathbf{0} \quad \text { on } \partial \Omega \text {. }
$$

Since Dirichlet boundary conditions are prescribed on the whole boundary, we fix the absolute value of the pressure by postulating the additional constraint

$$
\frac{1}{|\Omega|} \int_{\Omega} \pi \mathrm{d} \boldsymbol{x}=0
$$

This paper is dedicated to the finite element (FE) approximation of (1.1)-(1.5) in the case $p<2$. Optimal a priori error estimates quantifying the convergence of the finite element method (FEM) have recently been derived (see [15, 4]). They include the singular case $\varepsilon=0$. However, numerical methods solving the FE systems generally suffer from instabilities as soon as $\varepsilon \searrow 0$. The nonlinear discrete equations are frequently solved by means of Newton's method (cf. [10]). It can be seen easily that, in the singular case, the nonlinear operator associated with $\mathcal{S}(\boldsymbol{D} \boldsymbol{v})$ is not differentiable on the critical set $\{\boldsymbol{x} \in \Omega ; \nabla \boldsymbol{v}(\boldsymbol{x})=\mathbf{0}\}$. (Note that this set is not empty for typical solutions to (1.1)-(1.5).) Since Newton's method requires the existence of first derivatives, for $\varepsilon=0$ the FE solution cannot be determined via Newton's method in general. This paper aims at developing a numerical method which enables the stable approximation of singular power-law systems.

First, in the context of models (1.3) we discuss Newton's method and its stability. For the Carreau model $(\varepsilon>0)$ the associated operator is differentiable and Newton's method is stable in the sense that the matrix resulting from linearization of the viscous part remains positive definite for $\varepsilon>0$. In particular, we prove the upper bound $c(p-1)^{-1} \varepsilon^{p-2} h^{-2}$ for the condition number of this matrix. Then we propose an approximation scheme for singular power-law systems that generates a sequence of discrete functions which is computable in practice via Newton's method and which converges to the exact solution of the power-law system. To this end we show that the solutions $\left(\boldsymbol{v}^{\varepsilon}, \pi^{\varepsilon}\right)$ to the Carreau systems, whose dependence on $\varepsilon>0$ is highlighted by the additional superscript, approximate the solution $(\boldsymbol{v}, \pi)$ to the power-law system for diminishing $\varepsilon \searrow 0$. In particular, we establish an $\mathcal{O}\left(\varepsilon^{\frac{p}{2}}\right)$ bound for $\left(\boldsymbol{v}^{\varepsilon}-\boldsymbol{v}\right)$ in $\boldsymbol{W}^{1, p}(\Omega)$ as well as an $\mathcal{O}\left(\varepsilon^{p-1}\right)$ bound for $\left(\pi^{\varepsilon}-\pi\right)$ in $L^{p^{\prime}}(\Omega), p^{\prime}:=p /(p-1)$. As a result we obtain an approximation method for power-law systems whose convergence is quantified by the a priori error estimates

$$
\left\|\boldsymbol{v}-\boldsymbol{v}_{h}^{\varepsilon}\right\|_{1, p} \lesssim \varepsilon^{p / 2}+\left\|\boldsymbol{v}-\boldsymbol{v}_{h}\right\|_{1, p}, \quad\left\|\pi-\pi_{h}^{\varepsilon}\right\|_{p^{\prime}} \lesssim \varepsilon^{p-1}+\left\|\pi-\pi_{h}\right\|_{p^{\prime}} .
$$

Here, $\boldsymbol{v}_{h}$ denotes the FE solution for the singular power-law model $(\varepsilon=0)$, and $\boldsymbol{v}_{h}^{\varepsilon}$ represents the FE solution for the Carreau model $(\varepsilon>0)$. Note that, in general, $\boldsymbol{v}_{h}$ cannot be determined by means of Newton's method. In contrast, $\boldsymbol{v}_{h}^{\varepsilon}$ is computable 
via Newton's method. Concerning the discretization errors $(\boldsymbol{v}, \pi)-\left(\boldsymbol{v}_{h}, \pi_{h}\right)$, optimal a priori error estimates are well known for particular finite elements; see [4, 15]. We couple the regularization parameter $\varepsilon$ with the mesh size $h$ so that the error caused by the regularization is of at least the same order as the discretization error. Finally, we demonstrate numerically that the proposed regularized approximation method surpasses the nonregularized one regarding accuracy and numerical efficiency.

The paper is organized as follows: In Section 2, we recall basic properties of the extra stress tensor, and we introduce the weak formulation of (1.1)-(1.5) and its discretization. Section 3 deals with Newton's method and its numerical stability. In Section 4 we present our numerical method which enables the stable approximation of singular power-law systems. Finally, in Section 5 we illustrate the theoretical results by numerical experiments.

\section{Preliminaries}

We introduce the weak formulation of (1.1)-(1.5) and its discretization. To begin with, we clarify our notation and we state important properties of the stress tensor.

Notation. The set of all positive real numbers is denoted by $\mathbb{R}^{+}$. Let $\mathbb{R}_{0}^{+}:=$ $\mathbb{R}^{+} \cup\{0\}$. The Euclidean scalar product of two vectors $\boldsymbol{p}, \boldsymbol{q} \in \mathbb{R}^{d}$ is denoted by $\boldsymbol{p} \cdot \boldsymbol{q}$, the scalar product of $\boldsymbol{P}, \boldsymbol{Q} \in \mathbb{R}^{d \times d}$ is defined by $\boldsymbol{P}: \boldsymbol{Q}:=\sum_{i, j=1}^{d} P_{i j} Q_{i j}$. Let us set $|\boldsymbol{Q}|:=(\boldsymbol{Q}: \boldsymbol{Q})^{1 / 2}$. Often we use $c$ as a generic constant whose value may change from line to line but does not depend on important variables. We write $a \sim b$ if there exist constants $c, C>0$ independent of all relevant quantities such that $c b \leq a \leq C b$. Similarly, the notation $a \lesssim b$ stands for $a \leq C b$.

If $\omega \subset \mathbb{R}^{d}$ is a measurable set, then $|\omega|$ denotes its $d$-dimensional Lebesgue measure. For $\nu \in[1, \infty], L^{\nu}(\Omega)$ stands for the Lebesgue space and $W^{m, \nu}(\Omega)$ for the Sobolev space of order $m$. For $\nu>1$ we use the notation $W_{0}^{1, \nu}(\Omega)$ for the Sobolev space with vanishing traces on $\partial \Omega$. The $L^{\nu}(\omega)$-norm is denoted by $\|\cdot\|_{\nu ; \omega}$ and the $W^{m, \nu}(\omega)$-norm is denoted by $\|\cdot\|_{m, \nu ; \omega}$. The notation $(u, v)_{\omega}$ is used for the integral $\int_{\omega} u v \mathrm{~d} \boldsymbol{x}$. In the case of $\omega=\Omega$, we usually omit the index $\Omega$. Spaces of $\mathbb{R}^{d}$-valued functions are denoted with boldface type, though no distinction is made in the notation of norms and inner products; The norm in $\boldsymbol{W}^{m, \nu}(\Omega) \equiv\left[W^{m, \nu}(\Omega)\right]^{d}$ is given by $\|\boldsymbol{w}\|_{m, \nu}=\left(\sum_{1 \leq i \leq d} \sum_{0 \leq|\alpha| \leq m}\left\|\partial^{\alpha} w_{i}\right\|_{\nu}^{\nu}\right)^{1 / \nu}$, etc. We recall the Poincaré and generalized Korn inequality (see, e.g., [18]): For any $\nu \in(1, \infty)$ there holds

$$
\|\boldsymbol{w}\|_{1, \nu} \leq c_{1}\|\nabla \boldsymbol{w}\|_{\nu} \leq c_{2}\|\boldsymbol{D} \boldsymbol{w}\|_{\nu} \quad \forall \boldsymbol{w} \in \boldsymbol{W}_{0}^{1, \nu}(\Omega),
$$

where the constants $c_{1}, c_{2}>0$ only depend on $\nu$ and $\Omega$. Let $X, Y$ be Banach spaces. If a mapping $J: X \rightarrow Y$ is Gâteaux differentiable in $x \in X$, then $J^{\prime}(x)(h)$ is referred to as the Gâteaux-derivative of $J$ at $x$ evaluated in direction $h \in X$ :

$$
J^{\prime}(x)(h):=\left.\frac{\mathrm{d}}{\mathrm{d} t} J(x+t h)\right|_{t=0}:=\lim _{t \searrow 0} \frac{1}{t}[J(x+t h)-J(x)] .
$$

Similarly, if $a(x)(y)$ is a semi-linear form, then $a^{\prime}(x)(h, y)$ denotes its directional derivative.

Extra stress tensor. For ease of presentation, we only study power-law/Carreautype models (1.2)-(1.3). Such models are derived from a potential. Let $\mathbb{R}_{\mathrm{sym}}^{d \times d}:=$ 
$\left\{\boldsymbol{Q} \in \mathbb{R}^{d \times d} ; \boldsymbol{Q}=\boldsymbol{Q}^{\top}\right\}$. For $p \in(1, \infty)$ and $\varepsilon \geq 0$, we define the extra stress tensor $\mathcal{S}_{\varepsilon}: \mathbb{R}_{\mathrm{sym}}^{d \times d} \rightarrow \mathbb{R}_{\mathrm{sym}}^{d \times d}$ by means of a convex function $\Phi_{\varepsilon} \in C^{1}\left(\mathbb{R}_{0}^{+}\right)$as follows:

$$
\mathcal{S}_{\varepsilon}(\boldsymbol{Q}):=\Phi_{\varepsilon}^{\prime}(|\boldsymbol{Q}|) \frac{\boldsymbol{Q}}{|\boldsymbol{Q}|} \quad \forall \boldsymbol{Q} \in \mathbb{R}_{\mathrm{sym}}^{d \times d}, \quad \Phi_{\varepsilon}(t):=\int_{0}^{t}\left(\varepsilon^{2}+s^{2}\right)^{\frac{p-2}{2}} s \mathrm{~d} s .
$$

The additional subscript $\varepsilon_{\varepsilon}$ highlights the dependence on $\varepsilon$ which will be of relevance below. For $p$ and $\varepsilon$ as in (2.1), we introduce the function $\mathcal{F}_{\varepsilon}: \mathbb{R}_{\mathrm{sym}}^{d \times d} \rightarrow \mathbb{R}_{\mathrm{sym}}^{d \times d}$ by

$$
\mathcal{F}_{\varepsilon}(\boldsymbol{Q}):=\left(\varepsilon^{2}+|\boldsymbol{Q}|^{2}\right)^{\frac{p-2}{4}} \boldsymbol{Q} .
$$

The extra stress tensor $\mathcal{S}_{\varepsilon}$ is closely related to the function $\mathcal{F}_{\varepsilon}$ as depicted by Lemma 2.1. Although we will only deal with the shear thinning case later on, we formulate all results as general as possible.

Lemma 2.1. For $p \in(1, \infty), \varepsilon \in[0, \infty)$ let the extra stress tensor $\mathcal{S}_{\varepsilon}$ be given by (2.1) and let $\mathcal{F}_{\varepsilon}$ be defined by (2.2). Then, for all $\boldsymbol{P}, \boldsymbol{Q} \in \mathbb{R}_{\mathrm{sym}}^{d \times d}$ there holds

$$
\begin{aligned}
\left(\mathcal{S}_{\varepsilon}(\boldsymbol{P})-\mathcal{S}_{\varepsilon}(\boldsymbol{Q})\right):(\boldsymbol{P}-\boldsymbol{Q}) & \sim(\varepsilon+|\boldsymbol{P}|+|\boldsymbol{Q}|)^{p-2}|\boldsymbol{P}-\boldsymbol{Q}|^{2} \\
& \sim\left|\mathcal{F}_{\varepsilon}(\boldsymbol{P})-\mathcal{F}_{\varepsilon}(\boldsymbol{Q})\right|^{2} \\
\left|\mathcal{S}_{\varepsilon}(\boldsymbol{P})-\mathcal{S}_{\varepsilon}(\boldsymbol{Q})\right| & \sim(\varepsilon+|\boldsymbol{P}|+|\boldsymbol{Q}|)^{p-2}|\boldsymbol{P}-\boldsymbol{Q}| .
\end{aligned}
$$

All constants only depend on p. In particular, they are independent of $\varepsilon \geq 0$.

Proof. The lemma is proven in Diening/Ettwein [11].

Remark 2.2. In view of Lemma 2.1, for all $\boldsymbol{v}, \boldsymbol{w} \in \boldsymbol{W}^{1, p}(\Omega)$ there holds

$$
\left(\mathcal{S}_{\varepsilon}(\boldsymbol{D} \boldsymbol{v})-\mathcal{S}_{\varepsilon}(\boldsymbol{D} \boldsymbol{w}), \boldsymbol{D} \boldsymbol{v}-\boldsymbol{D} \boldsymbol{w}\right)_{\Omega} \sim\left\|\mathcal{F}_{\varepsilon}(\boldsymbol{D} \boldsymbol{v})-\mathcal{F}_{\varepsilon}(\boldsymbol{D} \boldsymbol{w})\right\|_{2}^{2}
$$

The distance suggested by the right-hand (or left-hand) side is referred to as the natural distance (cf. Belenki et al. 4]). It is well known that the natural distance is equivalent to the quasi-norm introduced by Barrett and Liu [2].

The next lemma indicates how the natural distance relates to the Sobolev-norm:

Lemma 2.3. For $p \in(1,2]$ and $\varepsilon \in[0, \infty)$ let the extra stress tensor $\mathcal{S}_{\varepsilon}$ be given by (2.1) and let $\mathcal{F}_{\varepsilon}$ be defined by (2.2). For all $\boldsymbol{v}, \boldsymbol{w} \in \boldsymbol{W}^{1, p}(\Omega)$ there holds

$$
\|\boldsymbol{D} \boldsymbol{v}-\boldsymbol{D} \boldsymbol{w}\|_{p}^{2} \leq c\left\|\mathcal{F}_{\varepsilon}(\boldsymbol{D} \boldsymbol{v})-\mathcal{F}_{\varepsilon}(\boldsymbol{D} \boldsymbol{w})\right\|_{2}^{2}\|\varepsilon+|\boldsymbol{D} \boldsymbol{v}|+|\boldsymbol{D} \boldsymbol{w}|\|_{p}^{2-p}
$$

where the constant $c$ only depends on $p$.

Proof. It holds that $\left|\mathcal{F}_{\varepsilon}(\boldsymbol{D} \boldsymbol{v})-\mathcal{F}_{\varepsilon}(\boldsymbol{D} \boldsymbol{w})\right|(\varepsilon+|\boldsymbol{D} \boldsymbol{v}|+|\boldsymbol{D} \boldsymbol{w}|)^{\frac{2-p}{2}} \sim|\boldsymbol{D} \boldsymbol{v}-\boldsymbol{D} \boldsymbol{w}|$ a.e. in $\Omega$ due to Lemma 2.1 Raising this to the power $p$, integrating the result over $\Omega$, and applying Hölder's inequality with $\frac{p}{2}+\frac{2-p}{2}=1$, we easily complete the proof.

Lemma 2.4. For $p \in(1,2]$ and $\varepsilon \in[0, \infty)$ let the extra stress tensor $\mathcal{S}_{\varepsilon}$ be given by (2.1) and let $\mathcal{F}_{\varepsilon}$ be defined by (2.2). For all $\boldsymbol{v}, \boldsymbol{w} \in \boldsymbol{W}^{1, p}(\Omega)$ there holds

$$
\left\|\mathcal{S}_{\varepsilon}(\boldsymbol{D} \boldsymbol{v})-\mathcal{S}_{\varepsilon}(\boldsymbol{D} \boldsymbol{w})\right\|_{p^{\prime}} \leq c\left\|\mathcal{F}_{\varepsilon}(\boldsymbol{D} \boldsymbol{v})-\mathcal{F}_{\varepsilon}(\boldsymbol{D} \boldsymbol{w})\right\|_{2}^{\frac{2}{p^{\prime}}}
$$

where the constant $c$ only depends on $p$.

Proof. It holds that $\left|\mathcal{S}_{\varepsilon}(\boldsymbol{D} \boldsymbol{v})-\mathcal{S}_{\varepsilon}(\boldsymbol{D} \boldsymbol{w})\right| \sim(\varepsilon+|\boldsymbol{D} \boldsymbol{v}|+|\boldsymbol{D} \boldsymbol{w}|)^{p-2}|\boldsymbol{D} \boldsymbol{v}-\boldsymbol{D} \boldsymbol{w}|$ a.e. in $\Omega$ due to Lemma 2.1. Raising this to the power $p^{\prime}$ and integrating the result over $\Omega$, in view of $p^{\prime} \geq 2$ we easily derive the desired estimate.

Below we always assume that the extra stress tensor $\mathcal{S}_{\varepsilon}$ is given by (2.1). 
Weak formulation. The natural spaces for the velocity and pressure are given by

$$
\begin{aligned}
& \mathcal{X}^{p}:=W_{0}^{1, p}(\Omega)^{d}, \quad \mathcal{V}^{p}:=\left\{\boldsymbol{w} \in \mathcal{X}^{p} ; \operatorname{div} \boldsymbol{w}=0 \text { a.e. in } \Omega\right\}, \\
& \mathcal{Q}^{p}:=L_{0}^{p^{\prime}}(\Omega):=\left\{q \in L^{p^{\prime}}(\Omega) ;(q, 1)_{\Omega}=0\right\} .
\end{aligned}
$$

As usual, $p^{\prime}:=p /(p-1)$. Then the weak formulation of system (1.1)-(1.5) reads:

$\left(\mathbf{P}^{\varepsilon}\right)$ : For given $\boldsymbol{f} \in\left(\mathcal{X}^{p}\right)^{*}$ find $\left(\boldsymbol{v}^{\varepsilon}, \pi^{\varepsilon}\right) \in \mathcal{X}^{p} \times \mathcal{Q}^{p}$ such that

$$
\begin{aligned}
& \left(\mathcal{S}_{\varepsilon}\left(\boldsymbol{D} \boldsymbol{v}^{\varepsilon}\right), \boldsymbol{D} \boldsymbol{w}\right)_{\Omega}-\left(\pi^{\varepsilon}, \operatorname{div} \boldsymbol{w}\right)_{\Omega}=\langle\boldsymbol{f}, \boldsymbol{w}\rangle \quad \forall \boldsymbol{w} \in \mathcal{X}^{p}, \\
& \quad\left(\operatorname{div} \boldsymbol{v}^{\varepsilon}, q\right)_{\Omega}=0 \quad \forall q \in \mathcal{Q}^{p} .
\end{aligned}
$$

We can reformulate Problem $\left(\mathbf{P}^{\varepsilon}\right)$ by using divergence-free trial functions in (2.5):

$\left(\mathbf{Q}^{\varepsilon}\right):$ For given $\boldsymbol{f} \in\left(\mathcal{X}^{p}\right)^{*}$ find $\boldsymbol{v}^{\varepsilon} \in \mathcal{V}^{p}$ such that

$$
\left(\mathcal{S}_{\varepsilon}\left(\boldsymbol{D} \boldsymbol{v}^{\varepsilon}\right), \boldsymbol{D} \boldsymbol{w}\right)_{\Omega}=\langle\boldsymbol{f}, \boldsymbol{w}\rangle \quad \forall \boldsymbol{w} \in \mathcal{V}^{p} .
$$

It is well known that the two formulations are equivalent and that they are wellposed. Using the theory of monotone operators, we can easily conclude that there exists a unique solution $\boldsymbol{v}^{\varepsilon} \in \mathcal{V}^{p}$ to Problem $\left(\mathbf{Q}^{\varepsilon}\right)$; cf. [4. Since $\mathcal{S}_{\varepsilon}$ is derived from the potential $\Phi_{\varepsilon}$, we can introduce a functional $\mathcal{J}_{\varepsilon}: \mathcal{X}^{p} \rightarrow \mathbb{R}$ associated with $\Phi_{\varepsilon}$ :

$$
\mathcal{J}_{\varepsilon}(\boldsymbol{u}):=\int_{\Omega} \Phi_{\varepsilon}(|\boldsymbol{D} \boldsymbol{u}|) \mathrm{d} \boldsymbol{x}-\langle\boldsymbol{f}, \boldsymbol{u}\rangle \quad \forall \boldsymbol{u} \in \mathcal{X}^{p} .
$$

It is easy to check that the functional $\mathcal{J}_{\varepsilon}$ is Gâteaux differentiable on $\boldsymbol{\mathcal { X }}^{p}$ and that its derivative is given by $\mathcal{J}_{\varepsilon}^{\prime}(\boldsymbol{u})(\boldsymbol{w})=\left(\mathcal{S}_{\varepsilon}(\boldsymbol{D} \boldsymbol{u}), \boldsymbol{D} \boldsymbol{w}\right)_{\Omega}-\langle\boldsymbol{f}, \boldsymbol{w}\rangle$ for all $\boldsymbol{u}, \boldsymbol{w} \in \boldsymbol{\mathcal { X }}^{p}$. Since the operator $\boldsymbol{u} \mapsto-\operatorname{div} \mathcal{S}_{\varepsilon}(\boldsymbol{D} \boldsymbol{u})$ is strictly monotone on $\mathcal{X}^{p}, \mathcal{J}_{\varepsilon}^{\prime}$ is strictly monotone on $\mathcal{X}^{p}$ and, hence, $\mathcal{J}_{\varepsilon}$ is strictly convex on $\mathcal{X}^{p}$. In addition, $\mathcal{J}_{\varepsilon}$ is coercive on $\boldsymbol{\mathcal { X }}^{p}$, i.e., $\mathcal{J}_{\varepsilon}(\boldsymbol{u}) \rightarrow \infty$ for $\|\boldsymbol{D} \boldsymbol{u}\|_{p} \rightarrow \infty$. Because $\mathcal{V}^{p}$ is a closed convex subset of $\mathcal{X}^{p}$, it follows that there exists a unique solution to the minimization problem:

$\left(\mathbf{M}^{\varepsilon}\right)$ : For given $\boldsymbol{f} \in\left(\mathcal{X}^{p}\right)^{*}$ find $\boldsymbol{v}^{\varepsilon} \in \mathcal{V}^{p}$ such that

$$
\mathcal{J}_{\varepsilon}\left(\boldsymbol{v}^{\varepsilon}\right) \leq \mathcal{J}_{\varepsilon}(\boldsymbol{w}) \quad \forall \boldsymbol{w} \in \mathcal{V}^{p} .
$$

Furthermore, $\left(\mathbf{M}^{\varepsilon}\right)$ is equivalent to $\left(\mathbf{Q}^{\varepsilon}\right)$, its Euler equation.

If we take $\boldsymbol{w} \in \mathcal{V}^{p} \subset \mathcal{X}^{p}$ in equation (2.5), we realize that $\boldsymbol{v}^{\varepsilon}$, the solution to $\left(\mathbf{Q}^{\varepsilon}\right) \equiv\left(\mathbf{M}^{\varepsilon}\right)$, is the unique $\boldsymbol{v}^{\varepsilon}$ solving $\left(\mathbf{P}^{\varepsilon}\right)$. In order to prove the existence of a unique pressure $\pi^{\varepsilon} \in \mathcal{Q}^{p}$, we recall that the pairing $\mathcal{X}^{p} \times \mathcal{Q}^{p}$ satisfies the "inf-sup" (or Babuška-Brezzi) condition. In fact, the following result is well known (see [1]): For any $\nu \in(1, \infty)$ there exists a positive constant $\beta(\nu)$ such that

$$
\inf _{q \in L_{0}^{\nu^{\prime}}(\Omega)} \sup _{\boldsymbol{w} \in \boldsymbol{W}_{0}^{1, \nu}(\Omega)} \frac{(q, \operatorname{div} \boldsymbol{w})_{\Omega}}{\|q\|_{\nu^{\prime}}\|\boldsymbol{w}\|_{1, \nu}} \geq \beta(\nu)>0
$$

with $\nu^{\prime}=\nu /(\nu-1)$. This implies the existence of a unique solution $\left(\boldsymbol{v}^{\varepsilon}, \pi^{\varepsilon}\right)$ to $\left(\mathbf{P}^{\varepsilon}\right)$. In particular, the mixed weak formulation $\left(\mathbf{P}^{\varepsilon}\right)$ is equivalent to $\left(\mathbf{Q}^{\varepsilon}\right) \equiv\left(\mathbf{M}^{\varepsilon}\right)$.

Lemma 2.5. There exists a unique solution $\left(\boldsymbol{v}^{\varepsilon}, \pi^{\varepsilon}\right) \in \mathcal{X}^{p} \times \mathcal{Q}^{p}$ to Problem $\left(\mathbf{P}^{\varepsilon}\right)$ satisfying the a priori estimate

$$
\left\|\boldsymbol{v}^{\varepsilon}\right\|_{1, p} \leq c_{1}\left(\|\boldsymbol{f}\|_{-1, p^{\prime}}^{\frac{1}{p-1}}+c_{2} \varepsilon\right),
$$

where $c_{1}>0$ only depends on $\Omega, p$ and $c_{2}=1$ if $p<2$ and $c_{2}=0$ otherwise. 
Proof. We prove the a priori estimate (2.11) which will play a crucial role in the subsequent analysis. We restrict ourselves to the proof of (2.11) for $p<2$ since we can derive (2.11) for $p \geq 2$ using exactly the same arguments. Setting $\boldsymbol{w}:=\boldsymbol{v}^{\varepsilon}$ in (2.7), applying Lemma 2.1 and Lemma 2.3, for $p<2$ we conclude that

$$
\left\langle\boldsymbol{f}, \boldsymbol{v}^{\varepsilon}\right\rangle=\left(\mathcal{S}\left(\boldsymbol{D} \boldsymbol{v}^{\varepsilon}\right), \boldsymbol{D} \boldsymbol{v}^{\varepsilon}\right)_{\Omega} \sim\left\|\mathcal{F}_{\varepsilon}\left(\boldsymbol{D} \boldsymbol{v}^{\varepsilon}\right)\right\|_{2}^{2} \geq c\left\|\boldsymbol{D} \boldsymbol{v}^{\varepsilon}\right\|_{p}^{2}\left(\varepsilon+\left\|\boldsymbol{D} \boldsymbol{v}^{\varepsilon}\right\|_{p}\right)^{p-2}
$$

for some $c=c(\Omega, p)>0$. In the case of $\left\|\boldsymbol{D} \boldsymbol{v}^{\varepsilon}\right\|_{p} \geq \varepsilon$, we deduce that $\left\|\boldsymbol{D} \boldsymbol{v}^{\varepsilon}\right\|_{p}^{2} \leq$ $c\left\langle\boldsymbol{f}, \boldsymbol{v}^{\varepsilon}\right\rangle\left\|\boldsymbol{D} \boldsymbol{v}^{\varepsilon}\right\|_{p}^{2-p} \leq c\|\boldsymbol{f}\|_{-1, p^{\prime}}\left\|\boldsymbol{D} \boldsymbol{v}^{\varepsilon}\right\|_{p}^{3-p}$. As a result, we arrive at (2.11).

Finite element discretization. For ease of exposition we assume that $\Omega$ is a polygonal/polyhedral domain. Following the literature such as [8, 9], we introduce a shape-regular decomposition $\mathbb{T}_{h}$ of $\Omega$ into $d$-dimensional simplices (or quadrilaterals/hexahedra) so that $\bar{\Omega}=\bigcup_{K \in \mathbb{T}_{h}} \bar{K}$. As usual, the symbol $h_{K}$ stands for the diameter of an element $K \in \mathbb{T}_{h}$; The mesh parameter $h$ represents the maximum diameter of the cells, i.e., $h:=\max \left\{h_{K} ; K \in \mathbb{T}_{h}\right\}$. Let $\mathcal{X}_{h}^{p}$ and $\mathcal{Q}_{h}^{p}$ be two appropriate finite element spaces defined on $\mathbb{T}_{h}$ that satisfy

$$
\boldsymbol{\mathcal { X }}_{h}^{p} \subset \mathcal{X}^{p} \cap \boldsymbol{W}^{1, \infty}(\Omega) \quad \text { and } \quad \mathcal{Q}_{h}^{p} \subset \mathcal{Q}^{p} \cap L^{\infty}(\Omega) .
$$

Note that the inclusions $\boldsymbol{\mathcal { X }}_{h}^{p} \subset \boldsymbol{W}^{1, \infty}(\Omega)$ and $\mathcal{Q}_{h}^{p} \subset L^{\infty}(\Omega)$ hold for all practical choices of $\mathcal{X}_{h}^{p}$ and $\mathcal{Q}_{h}^{p}$. The pure Galerkin approximation of $\left(\mathbf{P}^{\varepsilon}\right)$ consists in replacing the Banach spaces $\mathcal{X}^{p}, \mathcal{Q}^{p}$ by the finite dimensional spaces $\mathcal{X}_{h}^{p}, \mathcal{Q}_{h}^{p}$ :

$\left(\mathbf{P}_{h}^{\varepsilon}\right):$ Find $\left(\boldsymbol{v}_{h}^{\varepsilon}, \pi_{h}^{\varepsilon}\right) \in \mathcal{X}_{h}^{p} \times \mathcal{Q}_{h}^{p}$ such that

$$
\begin{aligned}
\left(\mathcal{S}_{\varepsilon}\left(\boldsymbol{D} \boldsymbol{v}_{h}^{\varepsilon}\right), \boldsymbol{D} \boldsymbol{w}_{h}\right)_{\Omega}-\left(\pi_{h}^{\varepsilon}, \operatorname{div} \boldsymbol{w}_{h}\right)_{\Omega}=\left\langle\boldsymbol{f}, \boldsymbol{w}_{h}\right\rangle \quad \forall \boldsymbol{w}_{h} \in \boldsymbol{\mathcal { X }}_{h}^{p}, \\
\left(\operatorname{div} \boldsymbol{v}_{h}^{\varepsilon}, q_{h}\right)_{\Omega}=0 \quad \forall q_{h} \in \mathcal{Q}_{h}^{p} .
\end{aligned}
$$

In order to ensure the well-posedness of $\left(\mathbf{P}_{h}^{\varepsilon}\right)$, we require a discrete version of (2.10):

(IS): For any $\nu \in(1, \infty)$ there exists a constant $\tilde{\beta}(\nu)$ independent of $h$ so that

$$
\inf _{q_{h} \in \mathcal{Q}_{h}^{\nu}} \sup _{\boldsymbol{w}_{h} \in \mathcal{X}_{h}^{\nu}} \frac{\left(q_{h}, \operatorname{div} \boldsymbol{w}_{h}\right)_{\Omega}}{\left\|q_{h}\right\|_{\nu^{\prime}}\left\|\boldsymbol{w}_{h}\right\|_{1, \nu}} \geq \tilde{\beta}(\nu)>0
$$

If (IS) holds, then the well-posedness of Problem $\left(\mathbf{P}_{h}^{\varepsilon}\right)$ can easily be verified:

Lemma 2.6. Let (IS) hold. Then, there exists a unique solution $\left(\boldsymbol{v}_{h}^{\varepsilon}, \pi_{h}^{\varepsilon}\right) \in \boldsymbol{X}_{h}^{p} \times$ $\mathcal{Q}_{h}^{p}$ to Problem $\left(\mathbf{P}_{h}^{\varepsilon}\right)$ that satisfies the a priori bound (2.11) with $\boldsymbol{v}^{\varepsilon}$ replaced by $\boldsymbol{v}_{h}^{\varepsilon}$.

Remark 2.7. If the pairing $\mathcal{X}_{h}^{p} \times \mathcal{Q}_{h}^{p}$ does not satisfy (IS), the discrete pressure $\pi_{h}^{\varepsilon}$ may become unbounded in $L^{p^{\prime}}(\Omega)$ as $h \searrow 0$ or it may exhibit oscillations which do not reflect the physical objectivity. In this case, we need to stabilize the Galerkin discretization $\left(\mathbf{P}_{h}^{\varepsilon}\right)$. Stabilization methods, that are frequently used, are the local projection stabilization (LPS) and the pressure-stabilization Petrov-Galerkin (PSPG) method (see [7); e.g., LPS is particularly designed for equal-order discretizations. In this case, both the velocity and pressure are discretized with finite elements based on piecewise polynomials of the same degree. It is known that such discretizations are not stable. In the case of LPS, one adds an appropriate stabilization term $s_{h}\left(\pi_{h}, q_{h}\right)$ to (2.14), which controls the fluctuations of the pressure-gradient. 
In order to ensure approximation properties, one clearly needs to specify the choice of the discrete spaces. Since approximation properties are not important for the purpose of the forthcoming section, we will discuss particular choices of the discrete spaces later on.

\section{Stability of Newton's method}

In this section we discuss Newton's method and its stability in the context of power-law/Carreau models. In particular, we derive an upper bound for the condition number of the matrix resulting from linearization of the viscous part.

Solution of the discrete problems. We deal with the numerical solution of the algebraic systems. Due to its nonlinear nature, the discrete system (2.13)-(2.14) needs to be linearized. Here, we apply Newton's method for linearization. In order to describe the algorithm, we define a semi-linear form $a_{\varepsilon}(\cdot)(\cdot)$ associated with $\mathcal{S}_{\varepsilon}$ :

$$
a_{\varepsilon}(\boldsymbol{v})(\boldsymbol{w}):=\left(\mathcal{S}_{\varepsilon}(\boldsymbol{D} \boldsymbol{v}), \boldsymbol{D} \boldsymbol{w}\right)_{\Omega} \quad \forall \boldsymbol{v}, \boldsymbol{w} \in \mathcal{X}^{p} .
$$

Formally, we can compute the Gâteaux-derivative of $a_{\varepsilon}$ in the direction $\boldsymbol{\xi} \in \mathcal{X}^{p}$ :

$$
\begin{aligned}
a_{\varepsilon}^{\prime}(\boldsymbol{v})(\boldsymbol{\xi}, \boldsymbol{w})= & \int_{\Omega}\left(\varepsilon^{2}+|\boldsymbol{D} \boldsymbol{v}|^{2}\right)^{\frac{p-2}{2}} \boldsymbol{D} \boldsymbol{\xi}: \boldsymbol{D} \boldsymbol{w} \mathrm{d} \boldsymbol{x} \\
& +(p-2) \int_{\Omega}\left(\varepsilon^{2}+|\boldsymbol{D} \boldsymbol{v}|^{2}\right)^{\frac{p-4}{2}}(\boldsymbol{D} \boldsymbol{v}: \boldsymbol{D} \boldsymbol{\xi})(\boldsymbol{D} \boldsymbol{v}: \boldsymbol{D} \boldsymbol{w}) \mathrm{d} \boldsymbol{x} .
\end{aligned}
$$

Furthermore, for all $(\boldsymbol{v}, \pi),(\boldsymbol{w}, q) \in \mathcal{X}^{p} \times \mathcal{Q}^{p}$ we introduce the semi-linear form

$$
A_{\varepsilon}((\boldsymbol{v}, \pi))((\boldsymbol{w}, q)):=a_{\varepsilon}(\boldsymbol{v})(\boldsymbol{w})-(\pi, \operatorname{div} \boldsymbol{w})_{\Omega}+(\operatorname{div} \boldsymbol{v}, q)_{\Omega} .
$$

In order to determine a solution $\boldsymbol{U}_{h}=\left(\boldsymbol{v}_{h}, \pi_{h}\right) \in \mathcal{X}_{h}^{p} \times \mathcal{Q}_{h}^{p}$ to the discrete system

$$
A_{\varepsilon}\left(\boldsymbol{U}_{h}\right)\left(\boldsymbol{W}_{h}\right)-\left\langle\boldsymbol{f}, \boldsymbol{w}_{h}\right\rangle=0 \quad \forall \boldsymbol{W}_{h}=\left(\boldsymbol{w}_{h}, q_{h}\right) \in \boldsymbol{\mathcal { X }}_{h}^{p} \times \mathcal{Q}_{h}^{p},
$$

we carry out Newton's algorithm with step-size control:

1: Choose an initial guess $\boldsymbol{U}_{h}^{0}=\left(\boldsymbol{v}_{h}^{0}, \pi_{h}^{0}\right) \in \mathcal{X}_{h}^{p} \times \mathcal{Q}_{h}^{p}$.

2: Compute $\boldsymbol{Z}_{h}^{k}=\left(\boldsymbol{\xi}_{h}^{k}, \eta_{h}^{k}\right) \in \mathcal{X}_{h}^{p} \times \mathcal{Q}_{h}^{p}, k=0,1, \ldots$, from

$$
A_{\varepsilon}^{\prime}\left(\boldsymbol{U}_{h}^{k}\right)\left(\boldsymbol{Z}_{h}^{k}, \boldsymbol{W}_{h}\right)=-A_{\varepsilon}\left(\boldsymbol{U}_{h}^{k}\right)\left(\boldsymbol{W}_{h}\right)+\left\langle\boldsymbol{f}, \boldsymbol{w}_{h}\right\rangle \quad \forall \boldsymbol{W}_{h} \in \mathcal{X}_{h}^{p} \times \mathcal{Q}_{h}^{p},
$$

where the directional derivative is given by

$$
A_{\varepsilon}^{\prime}\left(\boldsymbol{U}_{h}^{k}\right)\left(\boldsymbol{Z}_{h}^{k}, \boldsymbol{W}_{h}\right)=a_{\varepsilon}^{\prime}\left(\boldsymbol{v}_{h}^{k}\right)\left(\boldsymbol{\xi}_{h}^{k}, \boldsymbol{w}_{h}\right)-\left(\eta_{h}^{k}, \operatorname{div} \boldsymbol{w}_{h}\right)_{\Omega}+\left(\operatorname{div} \boldsymbol{\xi}_{h}^{k}, q_{h}\right)_{\Omega} .
$$

3: For given $\lambda \in(0,1)$ determine minimal $l=0,1, \ldots$, for which

$$
R\left(\boldsymbol{U}_{h, l}^{k+1}\right)<R\left(\boldsymbol{U}_{h}^{k}\right), \quad \boldsymbol{U}_{h, l}^{k+1}:=\boldsymbol{U}_{h}^{k}+\lambda^{l} \boldsymbol{Z}_{h}^{k},
$$

and denote it by $l^{*}$, where the nonlinear residual $R(\cdot)$ is defined by

$$
R\left(\boldsymbol{U}_{h}\right):=\max _{i, j}\left\{A_{\varepsilon}\left(\boldsymbol{U}_{h}\right)\left(\left(\boldsymbol{\psi}^{i}, \chi^{j}\right)\right)-\left\langle\boldsymbol{f}, \boldsymbol{\psi}^{i}\right\rangle\right\} \quad \forall \boldsymbol{U}_{h} \in \boldsymbol{\mathcal { X }}_{h}^{p} \times \mathcal{Q}_{h}^{p} .
$$

Here, $\left\{\boldsymbol{\psi}^{i}\right\}$ and $\left\{\chi^{j}\right\}$ is the nodal basis of $\boldsymbol{\mathcal { X }}_{h}^{p}$ and $\mathcal{Q}_{h}^{p}$, respectively.

4: Set $\boldsymbol{U}_{h}^{k+1}:=\boldsymbol{U}_{h, l^{*}}^{k+1}$.

For $\epsilon=0$ the Gâteaux-derivative $a_{\varepsilon}^{\prime}\left(\boldsymbol{v}_{h}\right)\left(\boldsymbol{\xi}_{h}, \boldsymbol{w}_{h}\right)$ does not exist in general when the set $\left\{\boldsymbol{x} \in \Omega ; \nabla \boldsymbol{v}_{h}(\boldsymbol{x})=\mathbf{0}\right\}$ is not empty. In this case, the solution to (3.2) cannot be determined by means of Newton's method in general. However, if $\varepsilon>0$, it can easily be shown that $a_{\varepsilon}$ is Gâteaux differentiable on $\mathcal{X}_{h}^{p} \times \mathcal{X}_{h}^{p}$. Step [3 includes 
the step-size control which is crucial when highly nonlinear $p$-structure problems are solved via Newton's method. In general, the Newton update $\boldsymbol{Z}_{h}^{k}$ is weighted by the relaxation parameter $\lambda^{l}$. The step-size control enables the globalization of Newton's method, i.e., the independence of the convergence with respect to the choice of $\boldsymbol{U}_{h}^{0}$. If $l^{*}=0$, then the algorithm performs one full Newton cycle.

Stability of Newton's method. First of all, we discuss the algebraic structure of Newton's algorithm. For simplicity, we assume that Problem $\left(\mathbf{P}^{\varepsilon}\right)$ is discretized with inf-sup stable finite elements. In this case, the Galerkin discretization (3.2) does not need to be modified by additional stabilization terms. If equalorder discretizations are considered (cf. Remark 2.7), then the forthcoming investigations can easily be generalized. Let $\left\{\boldsymbol{\psi}^{j}, j=1, \ldots, N:=\operatorname{dim}\left(\boldsymbol{\mathcal { X }}_{h}^{p}\right)\right\}$ and $\left\{\chi^{j}, j=1, \ldots, M:=\operatorname{dim}\left(\mathcal{Q}_{h}^{p}\right)\right\}$ be the nodal basis of the finite element space $\mathcal{X}_{h}^{p}$ and $\mathcal{Q}_{h}^{p}$, respectively. In view of the representations

$$
\boldsymbol{Z}_{h}^{k}=\left(\boldsymbol{\xi}_{h}^{k}, \eta_{h}^{k}\right), \quad \boldsymbol{\xi}_{h}^{k}=\sum_{j=1}^{N} \alpha_{j}^{k} \boldsymbol{\psi}^{j}, \quad \eta_{h}^{k}=\sum_{j=1}^{M} \beta_{j}^{k} \chi^{j},
$$

Newton's system (3.3) is equivalent to the linear system of equations

$$
\left(\begin{array}{cc}
\boldsymbol{A}^{k} & \boldsymbol{B} \\
-\boldsymbol{B}^{\top} & \mathbf{0}
\end{array}\right)\left(\begin{array}{c}
\boldsymbol{\alpha}^{k} \\
\boldsymbol{\beta}^{k}
\end{array}\right)=\left(\begin{array}{l}
\boldsymbol{b}^{k} \\
\boldsymbol{c}^{k}
\end{array}\right)
$$

for the unknowns $\boldsymbol{\alpha}^{k} \in \mathbb{R}^{N}$ and $\boldsymbol{\beta}^{k} \in \mathbb{R}^{M}$, where

$$
\begin{aligned}
\boldsymbol{A}^{k} & :=\left(a_{\varepsilon}^{\prime}\left(\boldsymbol{v}_{h}^{k}\right)\left(\boldsymbol{\psi}^{j}, \boldsymbol{\psi}^{i}\right)\right)_{i, j=1}^{N}, \quad \boldsymbol{B}:=-\left(\left(\chi^{j}, \nabla \cdot \boldsymbol{\psi}^{i}\right)_{\Omega}\right)_{i, j=1}^{N, M}, \\
\boldsymbol{b}^{k} & :=\left(-a_{\varepsilon}\left(\boldsymbol{v}_{h}^{k}\right)\left(\boldsymbol{\psi}^{i}\right)+\left(\pi_{h}^{k}, \operatorname{div} \boldsymbol{\psi}^{i}\right)_{\Omega}+\left\langle\boldsymbol{f}, \boldsymbol{\psi}^{i}\right\rangle\right)_{i=1}^{N}, \\
\boldsymbol{c}^{k} & :=\left(-\left(\operatorname{div} \boldsymbol{v}_{h}^{k}, \chi^{i}\right)_{\Omega}\right)_{i=1}^{M} .
\end{aligned}
$$

The following theorem provides an upper bound for the condition number of $\boldsymbol{A}^{k}$.

Theorem 3.1. Let $p \in(1,2], \varepsilon \in(0, \infty)$. Then, the matrix $\boldsymbol{A}^{k}$ defined in (3.6) is symmetric and positive definite for all $k \in \mathbb{N}$. Hence, the matrix $\boldsymbol{A}^{k}$ is regular for all $k \in \mathbb{N}$. Furthermore, its condition number $\operatorname{cond}_{2}\left(\boldsymbol{A}^{k}\right)$ can be estimated by

$$
\operatorname{cond}_{2}\left(\boldsymbol{A}^{k}\right):=\frac{\lambda_{\max }\left(\boldsymbol{A}^{k}\right)}{\lambda_{\min }\left(\boldsymbol{A}^{k}\right)} \leq c(p-1)^{-1}\left(\varepsilon+\left\|\boldsymbol{D} \boldsymbol{v}_{h}^{k}\right\|_{\infty}\right)^{2-p} \epsilon^{p-2} h^{-2} .
$$

Here, $\lambda_{\max }\left(\boldsymbol{A}^{k}\right)$ and $\lambda_{\min }\left(\boldsymbol{A}^{k}\right)$ denote the largest and smallest eigenvalue of $\boldsymbol{A}^{k}$. The constant $c$ only depends on $\Omega$ and the shape-regularity of the grid $\mathbb{T}_{h}$.

Proof. Clearly, the matrix $\boldsymbol{A}^{k}$ defined in (3.6) is symmetric. Let $\boldsymbol{w}_{h} \in \boldsymbol{\mathcal { X }}_{h}^{p}$ be an arbitrary finite element function with corresponding nodal vector $\boldsymbol{\zeta}=\left(\zeta_{i}\right)_{i=1}^{N} \in \mathbb{R}^{N}$, i.e., $\boldsymbol{w}_{h}=\sum_{i=1}^{N} \zeta_{i} \boldsymbol{\psi}^{i}$. Then, the following identity holds true:

$$
a_{\varepsilon}^{\prime}\left(\boldsymbol{v}_{h}^{k}\right)\left(\boldsymbol{w}_{h}, \boldsymbol{w}_{h}\right)=\sum_{i, j=1}^{N} \zeta_{i} a_{\varepsilon}^{\prime}\left(\boldsymbol{v}_{h}^{k}\right)\left(\boldsymbol{\psi}^{i}, \boldsymbol{\psi}^{j}\right) \zeta_{j}=\sum_{i, j=1}^{N} \zeta_{i} A_{i j}^{k} \zeta_{j}
$$


In view of (3.1), we observe that

$$
\begin{aligned}
a_{\varepsilon}^{\prime}\left(\boldsymbol{v}_{h}^{k}\right)\left(\boldsymbol{w}_{h}, \boldsymbol{w}_{h}\right)= & \int_{\Omega}\left(\varepsilon^{2}+\left|\boldsymbol{D} \boldsymbol{v}_{h}^{k}\right|^{2}\right)^{\frac{p-2}{2}}\left|\boldsymbol{D} \boldsymbol{w}_{h}\right|^{2} \mathrm{~d} \boldsymbol{x} \\
& +(p-2) \int_{\Omega}\left(\varepsilon^{2}+\left|\boldsymbol{D} \boldsymbol{v}_{h}^{k}\right|^{2}\right)^{\frac{p-4}{2}}\left|\boldsymbol{D} \boldsymbol{v}_{h}^{k}: \boldsymbol{D} \boldsymbol{w}_{h}\right|^{2} \mathrm{~d} \boldsymbol{x} .
\end{aligned}
$$

Due to $p<2$ we can estimate this quantity as follows:

$$
\begin{aligned}
a_{\varepsilon}^{\prime}\left(\boldsymbol{v}_{h}^{k}\right)\left(\boldsymbol{w}_{h}, \boldsymbol{w}_{h}\right) \geq & \int_{\Omega}\left(\varepsilon^{2}+\left|\boldsymbol{D} \boldsymbol{v}_{h}^{k}\right|^{2}\right)^{\frac{p-2}{2}}\left|\boldsymbol{D} \boldsymbol{w}_{h}\right|^{2} \mathrm{~d} \boldsymbol{x} \\
& \quad+(p-2) \int_{\Omega}\left(\varepsilon^{2}+\left|\boldsymbol{D} \boldsymbol{v}_{h}^{k}\right|^{2}\right)^{\frac{p-4}{2}}\left|\boldsymbol{D} \boldsymbol{v}_{h}^{k}\right|^{2}\left|\boldsymbol{D} \boldsymbol{w}_{h}\right|^{2} \mathrm{~d} \boldsymbol{x} \\
\geq & (p-1) \int_{\Omega}\left(\varepsilon^{2}+\left|\boldsymbol{D} \boldsymbol{v}_{h}^{k}\right|^{2}\right)^{\frac{p-2}{2}}\left|\boldsymbol{D} \boldsymbol{w}_{h}\right|^{2} \mathrm{~d} \boldsymbol{x} \\
\geq & (p-1)\left(\varepsilon+\left\|\boldsymbol{D} \boldsymbol{v}_{h}^{k}\right\|_{\infty}\right)^{p-2}\left\|\boldsymbol{D} \boldsymbol{w}_{h}\right\|_{2}^{2} .
\end{aligned}
$$

Using the Korn-Poincaré inequality, for some $c=c(\Omega)>0$ we arrive at

$$
\sum_{i, j=1}^{N} \zeta_{i} A_{i j}^{k} \zeta_{j} \geq c(p-1)\left(\varepsilon+\left\|\boldsymbol{D} \boldsymbol{v}_{h}^{k}\right\|_{\infty}\right)^{p-2}\left\|\boldsymbol{w}_{h}\right\|_{1,2}^{2}
$$

Hence, the matrix $\boldsymbol{A}^{k}$ is positive definite. Let $\boldsymbol{M}$ be the mass matrix associated with $\mathcal{X}_{h}^{p}$, i.e., let $\boldsymbol{M} \in \mathbb{R}^{N \times N}$ be defined by $M_{i j}:=\left(\boldsymbol{\psi}^{i}, \boldsymbol{\psi}^{j}\right)_{\Omega}$. Then, there holds

$$
\left\|\boldsymbol{w}_{h}\right\|_{2}^{2}=\sum_{i, j=1}^{N} \zeta_{i} M_{i j} \zeta_{j}, \quad \operatorname{cond}_{2}(\boldsymbol{M})=\mathcal{O}(1)
$$

The smallest eigenvalue $\lambda_{\min }\left(\boldsymbol{A}^{k}\right)$ is bounded from below by

$$
\begin{aligned}
& \lambda_{\min }\left(\boldsymbol{A}^{k}\right)=\min _{\boldsymbol{\zeta} \in \mathbb{R}^{N}} \frac{\sum_{i, j=1}^{N} \zeta_{i} A_{i j}^{k} \zeta_{j}}{|\boldsymbol{\zeta}|^{2}} \\
& \geq \min _{\boldsymbol{\zeta} \in \mathbb{R}^{N}} \frac{\sum_{i, j=1}^{N} \zeta_{i} A_{i j}^{k} \zeta_{j}}{\sum_{i, j=1}^{N} \zeta_{i} M_{i j} \zeta_{j}} \min _{\boldsymbol{\zeta} \in \mathbb{R}^{N}} \frac{\sum_{i, j=1}^{N} \zeta_{i} M_{i j} \zeta_{j}}{|\boldsymbol{\zeta}|^{2}}=\min _{\boldsymbol{\zeta} \in \mathbb{R}^{N}} \frac{\sum_{i, j=1}^{N} \zeta_{i} A_{i j}^{k} \zeta_{j}}{\sum_{i, j=1}^{N} \zeta_{i} M_{i j} \zeta_{j}} \lambda_{\min }(\boldsymbol{M}) .
\end{aligned}
$$

Using (3.9) and applying the Korn-Poincaré inequality, we conclude that

$$
\begin{aligned}
\lambda_{\min }\left(\boldsymbol{A}^{k}\right) & \geq \min _{\boldsymbol{w}_{h} \in \mathcal{X}_{h}^{p}} \frac{a_{\varepsilon}^{\prime}\left(\boldsymbol{v}_{h}^{k}\right)\left(\boldsymbol{w}_{h}, \boldsymbol{w}_{h}\right)}{\left\|\boldsymbol{w}_{h}\right\|_{2}^{2}} \lambda_{\min }(\boldsymbol{M}) \\
& \geq c(p-1)\left(\varepsilon+\left\|\boldsymbol{D} \boldsymbol{v}_{h}^{k}\right\|_{\infty}\right)^{p-2} \lambda_{\min }(\boldsymbol{M})
\end{aligned}
$$

for some $c=c(\Omega)>0$. Similarly, we get an upper bound for the largest eigenvalue:

$$
\lambda_{\max }\left(\boldsymbol{A}^{k}\right)=\max _{\boldsymbol{\zeta} \in \mathbb{R}^{N}} \frac{\sum_{i, j=1}^{N} \zeta_{i} A_{i j}^{k} \zeta_{j}}{|\boldsymbol{\zeta}|^{2}} \leq \max _{\zeta \in \mathbb{R}^{N}} \frac{\sum_{i, j=1}^{N} \zeta_{i} A_{i j}^{k} \zeta_{j}}{\sum_{i, j=1}^{N} \zeta_{i} M_{i j} \zeta_{j}} \lambda_{\max }(\boldsymbol{M}) .
$$

In view of $p<2$, it easily follows from (3.8) that

$$
a_{\varepsilon}^{\prime}\left(\boldsymbol{v}_{h}^{k}\right)\left(\boldsymbol{w}_{h}, \boldsymbol{w}_{h}\right) \leq \int_{\Omega}\left(\varepsilon^{2}+\left|\boldsymbol{D} \boldsymbol{v}_{h}^{k}\right|^{2}\right)^{\frac{p-2}{2}}\left|\boldsymbol{D} \boldsymbol{w}_{h}\right|^{2} \mathrm{~d} \boldsymbol{x} \leq \varepsilon^{p-2}\left\|\boldsymbol{D} \boldsymbol{w}_{h}\right\|_{2}^{2} .
$$

Furthermore, the inverse estimate for FE functions (see [9], Theorem 3.2.6),

$$
\left\|\nabla \boldsymbol{w}_{h}\right\|_{2}^{2} \leq C h^{-2}\left\|\boldsymbol{w}_{h}\right\|_{2}^{2} \quad \forall \boldsymbol{w}_{h} \in \mathcal{X}_{h}^{p},
$$


holds true in the case of quasi-uniform meshes. The constant $C>0$ only depends on the shape-regularity of $\mathbb{T}_{h}$. Thus, the largest eigenvalue of $\boldsymbol{A}^{k}$ can be estimated by

$$
\begin{aligned}
\lambda_{\max }\left(\boldsymbol{A}^{k}\right) & \leq \max _{\boldsymbol{w}_{h} \in \mathcal{X}_{h}^{p}} \frac{a_{\varepsilon}^{\prime}\left(\boldsymbol{v}_{h}^{k}\right)\left(\boldsymbol{w}_{h}, \boldsymbol{w}_{h}\right)}{\left\|\boldsymbol{w}_{h}\right\|_{2}^{2}} \lambda_{\max }(\boldsymbol{M}) \\
& \leq \frac{\epsilon^{p-2}\left\|\boldsymbol{D} \boldsymbol{w}_{h}\right\|_{2}^{2}}{\left\|\boldsymbol{w}_{h}\right\|_{2}^{2}} \lambda_{\max }(\boldsymbol{M}) \leq C \epsilon^{p-2} h^{-2} \lambda_{\max }(\boldsymbol{M})
\end{aligned}
$$

To sum up, we have proven that

$c(p-1)\left(\varepsilon+\left\|\boldsymbol{D} \boldsymbol{v}_{h}^{k}\right\|_{\infty}\right)^{p-2} \lambda_{\min }(\boldsymbol{M}) \leq \lambda_{\min }\left(\boldsymbol{A}^{k}\right) \leq \lambda_{\max }\left(\boldsymbol{A}^{k}\right) \leq C \epsilon^{p-2} h^{-2} \lambda_{\max }(\boldsymbol{M})$.

Using (3.10), we easily complete the proof.

Remark 3.2. For $p=2$, the result (3.7) is well known in the context of Stokes systems. If the elasticity problem is studied, i.e., if the pressure term and the constraint $\operatorname{div} \boldsymbol{v}=0$ are omitted, then the Newton matrix stated in (3.5) only consists of the bloc $\boldsymbol{A}^{k}$. Theorem 3.1 only yields an upper bound for the condition number of the matrix $\boldsymbol{A}^{k}$. However, numerical experiments indicate (cf. Example 1) that, indeed, the condition number of $\boldsymbol{A}^{k}$ can behave as the expression on the righthand side of (3.7). This means that, for diminishing $\epsilon>0$ and $p>1$, the condition number of $\boldsymbol{A}^{k}$ increases. Since $\boldsymbol{A}^{k}$ is regular for all $k \in \mathbb{N}$, one can eliminate the variable $\boldsymbol{\alpha}^{k}$ in the system of equations (3.5) so that $\boldsymbol{\beta}^{k}, \boldsymbol{\alpha}^{k}$ can be determined from

$$
\boldsymbol{B}^{\top}\left(\boldsymbol{A}^{k}\right)^{-1} \boldsymbol{B} \boldsymbol{\beta}^{k}=\boldsymbol{c}^{k}+\boldsymbol{B}^{\top}\left(\boldsymbol{A}^{k}\right)^{-1} \boldsymbol{b}^{k}, \quad \boldsymbol{\alpha}^{k}=\left(\boldsymbol{A}^{k}\right)^{-1}\left(\boldsymbol{b}^{k}-\boldsymbol{B} \boldsymbol{\beta}^{k}\right) .
$$

The matrix $\boldsymbol{B}^{\top}\left(\boldsymbol{A}^{k}\right)^{-1} \boldsymbol{B}$ is referred to as the "Schur complement". For its computation, one needs to determine the inverse matrix of $\boldsymbol{A}^{k}$. According to (3.7), the condition number of $\boldsymbol{A}^{k}$ can be large for $0<\varepsilon \ll 1$ and $p<2$. Hence, one has to construct appropriate preconditioning methods in order to solve (3.11) numerically.

Example 1. We intend to illustrate that $\operatorname{cond}_{2}\left(\boldsymbol{A}^{k}\right)$ can actually behave as $c(p) \varepsilon^{p-2}$ for fixed $h$; see Theorem 3.1. Here, we considered the one-dimensional $p$-Laplace equation $-\frac{\mathrm{d}}{\mathrm{d} x}\left(\left[\varepsilon^{2}+\left|v^{\prime}\right|^{2}\right]^{\frac{p-2}{2}} v^{\prime}\right)=1$ on $\Omega=(-1,1)$ complemented with the boundary conditions $v(-1)=v(+1)=0$. Note that for $\varepsilon=0$ the solution is given by $v(x)=\frac{1}{p^{\prime}}\left(1-|x|^{p /(p-1)}\right)$. We discretized the $p$-Laplace problem with piecewise linear finite elements. On a fixed grid with 128 elements, the discrete problems were solved by means of Newton's method for diminishing values of $\varepsilon$. In doing so, we determined the condition number $\operatorname{cond}_{2}(\boldsymbol{A})$ of the Newton matrix $\boldsymbol{A}=\boldsymbol{A}^{k^{*}}$, where $k^{*}$ denotes the iteration-index for which the Newton residual is sufficiently small. In Table 1 we depict the condition numbers $\operatorname{cond}_{2}(\boldsymbol{A})$ with respect to $\varepsilon$ for two values of $p$. We observe that, indeed, $\operatorname{cond}_{2}(\boldsymbol{A})$ behaves as $c(p) \varepsilon^{p-2}$. Moreover, we realize that $\operatorname{cond}_{2}(\boldsymbol{A})$ increases for diminishing $p$. We suppose that, in the higher-dimensional case, the condition numbers behave similarly and diverge for $\varepsilon \searrow 0$. Hence, we may expect numerical trouble when for $\varepsilon \approx 0$ the linear systems of equations (3.5) are solved via iterative methods without appropriate preconditioning. 
TABle 1. Development of $\operatorname{cond}_{2}(\boldsymbol{A})$ for diminishing $\epsilon$

\begin{tabular}{|c|c|c|c|c|}
\hline \multirow[b]{2}{*}{$\varepsilon$} & \multicolumn{2}{|c|}{$p=1.20$} & \multicolumn{2}{|c|}{$p=1.01$} \\
\hline & $\operatorname{cond}_{2}(\boldsymbol{A})$ & conv. & $\operatorname{cond}_{2}(\boldsymbol{A})$ & conv. \\
\hline $2^{-5}$ & $1.9568 \mathrm{e}+05$ & - & $6.2374 \mathrm{e}+05$ & - \\
\hline $2^{-6}$ & $3.4267 \mathrm{e}+05$ & - & $1.2380 \mathrm{e}+06$ & - \\
\hline $2^{-7}$ & $5.9707 \mathrm{e}+05$ & -0.80 & $2.4682 \mathrm{e}+06$ & -1.00 \\
\hline $2^{-8}$ & $1.0376 \mathrm{e}+06$ & -0.80 & $4.9193 \mathrm{e}+06$ & -1.00 \\
\hline $2^{-9}$ & $1.8007 \mathrm{e}+06$ & -0.80 & $9.7868 \mathrm{e}+06$ & -1.00 \\
\hline $2^{-10}$ & $3.1292 \mathrm{e}+06$ & -0.80 & $1.9458 \mathrm{e}+07$ & -1.00 \\
\hline expected & & -0.80 & & -0.99 \\
\hline
\end{tabular}

\section{Approximation of Singular POWER-LAW Systems}

This section is dedicated to the finite element approximation of power-law systems $(\varepsilon=0)$. As already mentioned, the nonlinear operator related to $\mathcal{S}_{\varepsilon}$ is not differentiable for $\varepsilon=0$ in the shear thinning case. The lack of differentiability may cause numerical instabilities when the nonlinear discrete systems are solved via Newton's method. In this section, we propose a numerical method that enables the stable approximation of singular power-law systems. The proposed method is based on a simple regularization of the power-law model.

Clearly, the Carreau model (1.3) with $\varepsilon>0$ can be interpreted as the regularized power-law model. Let the quantities $\mathcal{S}_{\varepsilon}, \mathcal{F}_{\varepsilon}, \Phi_{\varepsilon}, \mathcal{J}_{\varepsilon}$ be defined as in Section2 The subscript highlights the dependence on $\varepsilon$ which is of relevance for the subsequent analysis. Let us set $\mathcal{S}:=\mathcal{S}_{0}, \Phi:=\Phi_{0}, \mathcal{F}:=\mathcal{F}_{0}, \mathcal{J}:=\mathcal{J}_{0}$. As depicted by the following theorem, the solutions to the Carreau systems $(\varepsilon>0)$ approximate the solution to the power-law system $(\varepsilon=0)$ for diminishing $\varepsilon \searrow 0$.

Theorem 4.1. For $p \in(1,2)$ and $\epsilon \in\left[0, \varepsilon_{0}\right]$ let the extra stress tensor $\mathcal{S}_{\varepsilon}$ be given by (2.1) and let $\mathcal{F}_{\varepsilon}$ be defined by (2.2). For each $\epsilon \in\left[0, \varepsilon_{0}\right]$ let $\left(\boldsymbol{v}^{\varepsilon}, \pi^{\varepsilon}\right) \in \mathcal{X}^{p} \times \mathcal{Q}^{p}$ be the unique solution to $\left(\mathbf{P}^{\varepsilon}\right)$. Let $(\boldsymbol{v}, \pi):=\left(\boldsymbol{v}^{0}, \pi^{0}\right)$ denote the solution to the power-law system. Then, there hold the a priori estimates

$$
\begin{aligned}
\left\|\mathcal{F}_{\varepsilon}\left(\boldsymbol{D} \boldsymbol{v}^{\varepsilon}\right)-\mathcal{F}_{\varepsilon}(\boldsymbol{D} \boldsymbol{v})\right\|_{2} & \leq c(p, \Omega) \varepsilon^{p / 2} \\
\left\|\boldsymbol{D} \boldsymbol{v}^{\varepsilon}-\boldsymbol{D} \boldsymbol{v}\right\|_{p} & \leq c\left(p, \varepsilon_{0}, \Omega, \boldsymbol{f}\right) \epsilon^{p / 2} \\
\left\|\pi^{\varepsilon}-\pi\right\|_{p^{\prime}} & \leq c(\beta(p), p, \Omega) \varepsilon^{p-1},
\end{aligned}
$$

where the constants only depend on the quantities quoted within the brackets. In particular, $\left(\boldsymbol{v}^{\varepsilon}, \pi^{\varepsilon}\right)$ converges to $(\boldsymbol{v}, \pi)$ in $\mathcal{X}^{p} \times \mathcal{Q}^{p}$ strongly for $\epsilon \searrow 0$.

Proof. Since $\boldsymbol{v}^{\varepsilon}$ is the unique solution to $\left(\mathbf{P}^{\varepsilon}\right)$ for $\varepsilon \geq 0$, it can be characterized as the unique minimizer of the functional $\mathcal{J}_{\varepsilon}$ in $\mathcal{V}^{p}$, i.e., it satisfies

$$
\mathcal{J}_{\varepsilon}\left(\boldsymbol{v}^{\varepsilon}\right) \leq \mathcal{J}_{\varepsilon}(\boldsymbol{u}) \quad \forall \boldsymbol{u} \in \mathcal{V}^{p}
$$

for each $\varepsilon \geq 0$. Using the trivial inequality

$$
\Phi_{\varepsilon}(t)=\frac{1}{p}\left[\left(\varepsilon^{2}+t^{2}\right)^{p / 2}-\varepsilon^{p}\right] \leq \frac{1}{p} t^{p}=\Phi(t) \quad \forall t \in \mathbb{R}_{0}^{+},
$$


we conclude that $\mathcal{J}_{\epsilon}(\boldsymbol{u}) \leq \mathcal{J}(\boldsymbol{u})$ for all $\boldsymbol{u} \in \mathcal{V}^{p}$. We recall that $\boldsymbol{v} \in \mathcal{V}^{p}$ is the unique minimizer of $\mathcal{J}$. Consequently, we arrive at the inequalities

$$
\mathcal{J}_{\epsilon}(\boldsymbol{v}) \leq \mathcal{J}(\boldsymbol{v}) \leq \mathcal{J}\left(\boldsymbol{v}^{\varepsilon}\right)
$$

From the main theorem of calculus we deduce that

$$
\begin{aligned}
\mathcal{J}_{\epsilon}(\boldsymbol{v})-\mathcal{J}_{\epsilon}\left(\boldsymbol{v}^{\varepsilon}\right)= & \int_{0}^{1} \mathcal{J}_{\epsilon}^{\prime}\left(\boldsymbol{v}^{\varepsilon}+s\left(\boldsymbol{v}-\boldsymbol{v}^{\varepsilon}\right)\right)\left(\boldsymbol{v}-\boldsymbol{v}^{\varepsilon}\right) \mathrm{d} s \\
= & \int_{0}^{1}\left[\mathcal{J}_{\epsilon}^{\prime}\left(\boldsymbol{v}^{\varepsilon}+s\left(\boldsymbol{v}-\boldsymbol{v}^{\varepsilon}\right)\right)\left(\left[\boldsymbol{v}^{\varepsilon}+s\left(\boldsymbol{v}-\boldsymbol{v}^{\varepsilon}\right)\right]-\boldsymbol{v}^{\varepsilon}\right)\right. \\
& \left.\quad-\mathcal{J}_{\epsilon}^{\prime}\left(\boldsymbol{v}^{\varepsilon}\right)\left(\left[\boldsymbol{v}^{\varepsilon}+s\left(\boldsymbol{v}-\boldsymbol{v}^{\varepsilon}\right)\right]-\boldsymbol{v}^{\varepsilon}\right)\right] \frac{\mathrm{d} s}{s}+\mathcal{J}_{\epsilon}^{\prime}\left(\boldsymbol{v}^{\varepsilon}\right)\left(\boldsymbol{v}-\boldsymbol{v}^{\varepsilon}\right) \\
=: & I+\mathcal{J}_{\epsilon}^{\prime}\left(\boldsymbol{v}^{\varepsilon}\right)\left(\boldsymbol{v}-\boldsymbol{v}^{\varepsilon}\right) .
\end{aligned}
$$

Since $\boldsymbol{v}^{\varepsilon}$ is the minimizer of $\mathcal{J}_{\epsilon}$, the last term equals zero: $\mathcal{J}_{\epsilon}^{\prime}\left(\boldsymbol{v}^{\varepsilon}\right)\left(\boldsymbol{v}-\boldsymbol{v}^{\varepsilon}\right)=0$. Let us estimate the term $I$. On the one hand, inequality (4.4) implies that

$$
\begin{aligned}
I & =\mathcal{J}_{\epsilon}(\boldsymbol{v})-\mathcal{J}_{\epsilon}\left(\boldsymbol{v}^{\varepsilon}\right) \leq \mathcal{J}\left(\boldsymbol{v}^{\varepsilon}\right)-\mathcal{J}_{\epsilon}\left(\boldsymbol{v}^{\varepsilon}\right) \\
& =\int_{\Omega} \frac{1}{p}\left|\boldsymbol{D} \boldsymbol{v}^{\varepsilon}\right|^{p} \mathrm{~d} \boldsymbol{x}-\int_{\Omega} \frac{1}{p}\left[\left(\epsilon^{2}+\left|\boldsymbol{D} \boldsymbol{v}^{\varepsilon}\right|^{2}\right)^{p / 2}-\epsilon^{p}\right] \mathrm{d} \boldsymbol{x} \\
& =\frac{1}{p} \int_{\Omega}\left[\left|\boldsymbol{D} \boldsymbol{v}^{\varepsilon}\right|^{p}-\left(\epsilon^{2}+\left|\boldsymbol{D} \boldsymbol{v}^{\varepsilon}\right|^{2}\right)^{p / 2}\right] \mathrm{d} \boldsymbol{x}+\frac{|\Omega|}{p} \epsilon^{p} \leq \frac{|\Omega|}{p} \epsilon^{p} .
\end{aligned}
$$

On the other hand, Lemma 2.1 implies that

$$
\begin{aligned}
I & =\int_{0}^{1} \int_{\Omega}\left(\mathcal{S}_{\varepsilon}\left(\boldsymbol{D} \boldsymbol{v}^{\varepsilon}+s\left(\boldsymbol{D} \boldsymbol{v}-\boldsymbol{D} \boldsymbol{v}^{\varepsilon}\right)\right)-\mathcal{S}_{\varepsilon}\left(\boldsymbol{D} \boldsymbol{v}^{\varepsilon}\right)\right) \\
& \times\left(\left[\boldsymbol{D} \boldsymbol{v}^{\varepsilon}+s\left(\boldsymbol{D} \boldsymbol{v}-\boldsymbol{D} \boldsymbol{v}^{\varepsilon}\right)\right]-\boldsymbol{D} \boldsymbol{v}^{\varepsilon}\right) \mathrm{d} \boldsymbol{x} \frac{\mathrm{d} s}{s} \\
& \sim \int_{0}^{1} \int_{\Omega}\left(\varepsilon+\left|\boldsymbol{D} \boldsymbol{v}^{\varepsilon}+s \boldsymbol{D}\left(\boldsymbol{v}-\boldsymbol{v}^{\varepsilon}\right)\right|+\left|\boldsymbol{D} \boldsymbol{v}^{\varepsilon}\right|\right)^{p-2} s\left|\boldsymbol{D}\left(\boldsymbol{v}-\boldsymbol{v}^{\varepsilon}\right)\right|^{2} \mathrm{~d} \boldsymbol{x} \mathrm{d} s \\
& \sim \int_{0}^{1} \int_{\Omega}\left(\varepsilon+\left|\boldsymbol{D} \boldsymbol{v}^{\varepsilon}+s\right| \boldsymbol{D}\left(\boldsymbol{v}-\boldsymbol{v}^{\varepsilon}\right) \mid\right)^{p-2} s\left|\boldsymbol{D}\left(\boldsymbol{v}-\boldsymbol{v}^{\varepsilon}\right)\right|^{2} \mathrm{~d} \boldsymbol{x} \mathrm{d} s \\
& \sim \int_{\Omega}\left(\varepsilon+\left|\boldsymbol{D} \boldsymbol{v}^{\varepsilon}\right|+\left|\boldsymbol{D}\left(\boldsymbol{v}-\boldsymbol{v}^{\varepsilon}\right)\right|\right)^{p-2}\left|\boldsymbol{D}\left(\boldsymbol{v}-\boldsymbol{v}^{\varepsilon}\right)\right|^{2} \mathrm{~d} \boldsymbol{x} \\
& \sim\left\|\mathcal{F}_{\varepsilon}(\boldsymbol{D} \boldsymbol{v})-\mathcal{F}_{\varepsilon}\left(\boldsymbol{D} \boldsymbol{v}^{\varepsilon}\right)\right\|_{2}^{2},
\end{aligned}
$$

where the constants only depend on $p$. This inequality together with the upper bound (4.5) yields the desired estimate (4.1). Using Lemma 2.3, we conclude that

$$
\left\|\boldsymbol{D} \boldsymbol{v}^{\varepsilon}-\boldsymbol{D} \boldsymbol{v}\right\|_{p} \leq c\left\|\mathcal{F}_{\varepsilon}(\boldsymbol{D} \boldsymbol{v})-\mathcal{F}_{\varepsilon}\left(\boldsymbol{D} \boldsymbol{v}^{\varepsilon}\right)\right\|_{2}\left\|\epsilon+|\boldsymbol{D} \boldsymbol{v}|+\left|\boldsymbol{D} \boldsymbol{v}^{\varepsilon}\right|\right\|_{p^{\frac{2-p}{2}}}
$$

where the constant $c$ only depends on $p$. Hence, in view of (4.1) the inequality

$$
\left\|\boldsymbol{D} \boldsymbol{v}^{\varepsilon}-\boldsymbol{D} \boldsymbol{v}\right\|_{p} \leq c\left(\epsilon_{0}|\Omega|^{\frac{1}{p}}+\|\boldsymbol{D} \boldsymbol{v}\|_{p}+\left\|\boldsymbol{D} \boldsymbol{v}^{\varepsilon}\right\|_{p}\right)^{\frac{2-p}{2}} \epsilon^{p / 2}
$$

follows. Due to the a priori bound (2.11), the expression within the brackets is uniformly bounded by a constant only depending on $p, \varepsilon_{0}, \Omega$ and $\boldsymbol{f}$. This proves 
(4.2). In order to show (4.3), we recall that the functions $(\boldsymbol{v}, \pi)$ and $\left(\boldsymbol{v}^{\varepsilon}, \pi^{\varepsilon}\right)$ satisfy

$$
\begin{array}{r}
(\mathcal{S}(\boldsymbol{D} \boldsymbol{v}), \boldsymbol{D} \boldsymbol{w})_{\Omega}-(\pi, \operatorname{div} \boldsymbol{w})_{\Omega}=\langle\boldsymbol{f}, \boldsymbol{w}\rangle \\
\left(\mathcal{S}_{\varepsilon}\left(\boldsymbol{D} \boldsymbol{v}^{\varepsilon}\right), \boldsymbol{D} \boldsymbol{w}\right)_{\Omega}-\left(\pi^{\varepsilon}, \operatorname{div} \boldsymbol{w}\right)_{\Omega}=\langle\boldsymbol{f}, \boldsymbol{w}\rangle
\end{array}
$$

for all $\boldsymbol{w} \in \mathcal{X}^{p}$. Subtracting these equations, we immediately conclude that

$$
\left(\pi-\pi^{\varepsilon}, \operatorname{div} \boldsymbol{w}\right)_{\Omega}=\left(\mathcal{S}(\boldsymbol{D} \boldsymbol{v})-\mathcal{S}_{\varepsilon}\left(\boldsymbol{D} \boldsymbol{v}^{\varepsilon}\right), \boldsymbol{D} \boldsymbol{w}\right)_{\Omega} \quad \forall \boldsymbol{w} \in \mathcal{X}^{p}
$$

Using the inf-sup inequality (2.10) for $\mathcal{X}^{p} \times \mathcal{Q}^{p}$ and the identity (4.6), we deduce

$$
\begin{aligned}
& \beta\left\|\pi-\pi^{\varepsilon}\right\|_{p^{\prime}} \leq \sup _{\boldsymbol{w} \in \mathcal{X}^{p}} \frac{\left(\pi-\pi^{\varepsilon}, \operatorname{div} \boldsymbol{w}\right)_{\Omega}}{\|\boldsymbol{w}\|_{1, p}}=\sup _{\boldsymbol{w} \in \mathcal{X}^{p}} \frac{\left(\mathcal{S}(\boldsymbol{D} \boldsymbol{v})-\mathcal{S}_{\varepsilon}\left(\boldsymbol{D} \boldsymbol{v}^{\varepsilon}\right), \boldsymbol{D} \boldsymbol{w}\right)_{\Omega}}{\|\boldsymbol{w}\|_{1, p}} \\
& \leq\left\|\mathcal{S}(\boldsymbol{D} \boldsymbol{v})-\mathcal{S}_{\varepsilon}(\boldsymbol{D} \boldsymbol{v})\right\|_{p^{\prime}}+\left\|\mathcal{S}_{\varepsilon}(\boldsymbol{D} \boldsymbol{v})-\mathcal{S}_{\varepsilon}\left(\boldsymbol{D} \boldsymbol{v}^{\varepsilon}\right)\right\|_{p^{\prime}}=: J_{1}+J_{2} .
\end{aligned}
$$

In order to estimate $J_{1}$, we have to control $\left|\Phi^{\prime}(t)-\Phi_{\varepsilon}^{\prime}(t)\right|$. We recall that

$$
\left.|| a\right|^{q}-|b|^{q}\left|\sim(|a|+|b|)^{q-1}\right| a-b \mid \quad \forall a, b \in \mathbb{R}
$$

for each $q>0$ (cf. Lemma 2.1). Applying (4.8) with $q:=\frac{2-p}{2}$, we conclude that

$$
\begin{aligned}
\left|\Phi_{\varepsilon}^{\prime}(t)-\Phi^{\prime}(t)\right| & =\left(\varepsilon^{2}+t^{2}\right)^{\frac{p-2}{2}} t\left|1-\left(\frac{\varepsilon^{2}+t^{2}}{t^{2}}\right)^{\frac{2-p}{2}}\right| \\
& \sim\left(\varepsilon^{2}+t^{2}\right)^{\frac{p-2}{2}} t\left(1+\frac{\varepsilon^{2}+t^{2}}{t^{2}}\right)^{-\frac{p}{2}} \frac{\varepsilon^{2}}{t^{2}} \\
& \sim\left(\varepsilon^{2}+t^{2}\right)^{\frac{p-2}{2}}\left(\varepsilon^{2}+2 t^{2}\right)^{-\frac{p}{2}} t^{p-1} \varepsilon^{2} \sim t^{p-1} \frac{\varepsilon^{2}}{\varepsilon^{2}+t^{2}}
\end{aligned}
$$

uniformly in $t \in \mathbb{R}_{0}^{+}$(we used $\varepsilon^{2}+t^{2} \sim \varepsilon^{2}+2 t^{2}$ ). In particular, (4.9) implies that $\left|\Phi_{\varepsilon}^{\prime}(t)-\Phi^{\prime}(t)\right| \lesssim\left(\varepsilon^{2}+t^{2}\right)^{\frac{p-3}{2}} \varepsilon^{2} \lesssim \varepsilon^{p-1}$ uniformly in $t \in \mathbb{R}_{0}^{+}$. Thus, we obtain

$$
J_{1} \leq\left(\int_{\Omega}\left|\Phi^{\prime}(|\boldsymbol{D} \boldsymbol{v}|)-\Phi_{\varepsilon}^{\prime}(|\boldsymbol{D} \boldsymbol{v}|)\right|^{p^{\prime}} \mathrm{d} \boldsymbol{x}\right)^{\frac{1}{p^{\prime}}} \leq c|\Omega|^{\frac{1}{p^{\prime}}} \epsilon^{p-1},
$$

where the constant $c$ only depends on $p$. It remains to estimate the term $J_{2}$. Using Lemma 2.4 and inequality (4.1), we deduce that

$$
J_{2} \leq c\left\|\mathcal{F}_{\varepsilon}(\boldsymbol{D} \boldsymbol{v})-\mathcal{F}_{\varepsilon}\left(\boldsymbol{D} \boldsymbol{v}^{\varepsilon}\right)\right\|_{2}^{\frac{2}{p^{\prime}}} \leq c \varepsilon^{\frac{p 2}{2 p^{\prime}}}=c \varepsilon^{p-1}
$$

where the constant $c$ only depends on $p$ and $\Omega$. Combining (4.7), (4.10), and (4.11), we arrive at the desired result (4.3).

Remark 4.2. One can also derive error estimates similar to those in Theorem 4.1 without using the minimization property of the energy functional. Since $\boldsymbol{v}^{\varepsilon}$ are the solutions to Problem $\left(\mathbf{P}^{\varepsilon}\right)$ for $\varepsilon \geq 0$, they satisfy the equations

$$
\left(\mathcal{S}_{\varepsilon}\left(\boldsymbol{D} \boldsymbol{v}^{\varepsilon}\right), \boldsymbol{D} \boldsymbol{w}\right)_{\Omega}=\langle\boldsymbol{f}, \boldsymbol{w}\rangle \quad \text { and } \quad(\mathcal{S}(\boldsymbol{D} \boldsymbol{v}), \boldsymbol{D} \boldsymbol{w})_{\Omega}=\langle\boldsymbol{f}, \boldsymbol{w}\rangle
$$


for all $\boldsymbol{w} \in \mathcal{V}^{p}$. Subtracting the latter equations, we immediately conclude that $\left(\mathcal{S}_{\varepsilon}\left(\boldsymbol{D} \boldsymbol{v}^{\varepsilon}\right)-\mathcal{S}(\boldsymbol{D} \boldsymbol{v}), \boldsymbol{D} \boldsymbol{w}\right)_{\Omega}=0$ for all $\boldsymbol{w} \in \mathcal{V}^{p}$. By means of (4.9) we deduce that

$$
\begin{aligned}
\left\|\mathcal{F}\left(\boldsymbol{D} \boldsymbol{v}^{\varepsilon}\right)-\mathcal{F}(\boldsymbol{D} \boldsymbol{v})\right\|_{2}^{2} & \sim\left(\mathcal{S}\left(\boldsymbol{D} \boldsymbol{v}^{\varepsilon}\right)-\mathcal{S}(\boldsymbol{D} \boldsymbol{v}), \boldsymbol{D} \boldsymbol{v}^{\varepsilon}-\boldsymbol{D} \boldsymbol{v}\right)_{\Omega} \\
& =\left(\mathcal{S}\left(\boldsymbol{D} \boldsymbol{v}^{\varepsilon}\right)-\mathcal{S}_{\varepsilon}\left(\boldsymbol{D} \boldsymbol{v}^{\varepsilon}\right), \boldsymbol{D} \boldsymbol{v}^{\varepsilon}-\boldsymbol{D} \boldsymbol{v}\right)_{\Omega} \\
& =\int_{\Omega}\left[\Phi^{\prime}\left(\left|\boldsymbol{D} \boldsymbol{v}^{\varepsilon}\right|\right)-\Phi_{\varepsilon}^{\prime}\left(\left|\boldsymbol{D} \boldsymbol{v}^{\varepsilon}\right|\right)\right] \frac{\boldsymbol{D} \boldsymbol{v}^{\varepsilon}}{\left|\boldsymbol{D} \boldsymbol{v}^{\varepsilon}\right|}:\left(\boldsymbol{D} \boldsymbol{v}^{\varepsilon}-\boldsymbol{D} \boldsymbol{v}\right) \mathrm{d} \boldsymbol{x} \\
& \sim \varepsilon^{p-1} \int_{\Omega} \frac{\varepsilon^{3-p}\left|\boldsymbol{D} \boldsymbol{v}^{\varepsilon}\right|^{p-1}}{\varepsilon^{2}+\left|\boldsymbol{D} \boldsymbol{v}^{\varepsilon}\right|^{2}} \frac{\boldsymbol{D} \boldsymbol{v}^{\varepsilon} \mid}{\mid \boldsymbol{D} \boldsymbol{v}^{\varepsilon}}:\left(\boldsymbol{D} \boldsymbol{v}^{\varepsilon}-\boldsymbol{D} \boldsymbol{v}\right) \mathrm{d} \boldsymbol{x} .
\end{aligned}
$$

The real function $g(t):=\frac{\varepsilon^{3-p} t^{p-1}}{\varepsilon^{2}+t^{2}}, t \in \mathbb{R}_{0}^{+}$, is bounded by one:

$$
g(t) \leq\left\{\begin{array}{ll}
\frac{\varepsilon^{2}}{\varepsilon^{2}+t^{2}} & \text { if } t \leq \varepsilon \\
\frac{t^{2}}{\varepsilon^{2}+t^{2}} & \text { if } t>\varepsilon
\end{array} \quad \Rightarrow \quad g(t) \leq 1 \forall t \in \mathbb{R}_{0}^{+} .\right.
$$

Therefore, the integral in (4.12) is well defined. Using the continuous embedding $L^{p}(\Omega) \hookrightarrow L^{1}(\Omega)$, Lemma 2.3 , and the a priori bound (2.11), we finally arrive at

$$
\left\|\mathcal{F}\left(\boldsymbol{D} \boldsymbol{v}^{\varepsilon}\right)-\mathcal{F}(\boldsymbol{D} \boldsymbol{v})\right\|_{2} \leq c \varepsilon^{p-1},
$$

where the constant $c$ only depends on $p, \varepsilon_{0}, \Omega, \boldsymbol{f}$.

Application to the FE approximation. On the basis of Theorem 4.1 we now construct our approximation scheme for singular power-law systems. For this, we require certain approximation properties of the finite element method. For particular finite elements, the following result is well known (see [4, 15]): For $p \in(1,2]$ and $\varepsilon \in\left[0, \varepsilon_{0}\right]$ let $\left(\boldsymbol{v}^{\varepsilon}, \pi^{\varepsilon}\right)$ be the solution to $\left(\mathbf{P}^{\varepsilon}\right)$ and let $\left(\boldsymbol{v}_{h}^{\varepsilon}, \pi_{h}^{\varepsilon}\right)$ be the solution to $\left(\mathbf{P}_{h}^{\varepsilon}\right)$. If the solution $\left(\boldsymbol{v}^{\varepsilon}, \pi^{\varepsilon}\right)$ satisfies the regularity condition $\mathcal{F}_{\varepsilon}\left(\boldsymbol{D} \boldsymbol{v}^{\varepsilon}\right) \in W^{1,2}(\Omega) d \times d$ and $\pi^{\varepsilon} \in W^{1, p^{\prime}}(\Omega)$, then the discretization error can be estimated in terms of the maximum mesh size $h$ as follows:

$$
\left\|\mathcal{F}_{\varepsilon}\left(\boldsymbol{D} \boldsymbol{v}^{\varepsilon}\right)-\mathcal{F}_{\varepsilon}\left(\boldsymbol{D} \boldsymbol{v}_{h}^{\varepsilon}\right)\right\|_{2} \leq C_{1} h, \quad \tilde{\beta}(p)\left\|\pi^{\varepsilon}-\pi_{h}^{\varepsilon}\right\|_{p^{\prime}} \leq C_{2} h^{\frac{2}{p^{\prime}}} .
$$

The constants $C_{1}$ and $C_{2}$ depend on $\left\|\nabla \mathcal{F}_{\varepsilon}\left(\boldsymbol{D} \boldsymbol{v}^{\varepsilon}\right)\right\|_{2},\left\|\pi^{\varepsilon}\right\|_{1, p^{\prime}}, p, \varepsilon_{0}, \Omega$, and $\boldsymbol{f}$. In particular, they do not depend on $\varepsilon$ explicitly. Note that (4.13) remains valid for $\varepsilon=$ 0 . The error estimates (4.13) have been proven for various finite elements by [4, 15. In particular, in [4 they are derived for inf-sup stable finite elements based on $d$ simplices such as: $\mathbb{P}_{2} / \mathbb{P}_{0}$, Crouzeix-Raviart $\left(\mathbb{P}_{2}\right.$ plus bubble / discontinuous $\left.\mathbb{P}_{1}\right)$, MINI-element $\left(\mathbb{P}_{1}\right.$ plus bubble $\left./ \mathbb{P}_{1}\right)$. In $[15$, the error estimates $[4.13)$ are proven for equal-order $d$-linear finite elements $\left(\mathbb{Q}_{1} / \mathbb{Q}_{1}\right)$ based on quadrilateral meshes provided that the discrete equations are stabilized by a certain modified LPS method (see [15]). Note that (4.13) provides optimal convergence rates with respect to the supposed regularity of the solution. The following corollary is a simple consequence of Theorem 4.1. It yields the desired approximation scheme for singular power-law systems. Our approximation method generates a sequence of discrete functions $\left\{\boldsymbol{v}_{h}^{\varepsilon}\right\}$ which is computable in practice via Newton's method and which converges to the exact solution of the power-law system, $(\boldsymbol{v}, \pi)$.

Corollary 4.3. Let $p \in(1,2)$ and $\epsilon \in\left[0, \varepsilon_{0}\right]$. For each $\epsilon$ let $\left(\boldsymbol{v}^{\varepsilon}, \pi^{\varepsilon}\right) \in \mathcal{X}^{p} \times \mathcal{Q}^{p}$ be the unique solution to $\left(\mathbf{P}^{\varepsilon}\right)$, and let $\left(\boldsymbol{v}_{h}^{\varepsilon}, \pi_{h}^{\varepsilon}\right) \in \mathcal{X}_{h}^{p} \times \mathcal{Q}_{h}^{p}$ be the unique solution to $\left(\mathbf{P}_{h}^{\varepsilon}\right)$. Let us set $(\boldsymbol{v}, \pi):=\left(\boldsymbol{v}^{0}, \pi^{0}\right)$ and $\left(\boldsymbol{v}_{h}, \pi_{h}\right):=\left(\boldsymbol{v}_{h}^{0}, \pi_{h}^{0}\right)$. We suppose that $(\mathbf{I S})$ 
is satisfied. Furthermore, we assume that $(\boldsymbol{v}, \pi)$ satisfies the regularity condition

$$
\mathcal{F}(\boldsymbol{D} \boldsymbol{v}) \in W^{1,2}(\Omega)^{d \times d}, \quad \pi \in W^{1, p^{\prime}}(\Omega),
$$

and that the estimates (4.13) hold true for $\varepsilon=0$. Then, the solution to the powerlaw system $(\boldsymbol{v}, \pi)$ is approximated by the discrete functions $\left(\boldsymbol{v}_{h}^{\varepsilon}, \pi_{h}^{\varepsilon}\right)$ as $\varepsilon, h \searrow 0$ :

$$
\left\|\boldsymbol{v}-\boldsymbol{v}_{h}^{\varepsilon}\right\|_{1, p} \leq C_{1}\left(\varepsilon^{p / 2}+h\right), \quad\left\|\pi-\pi_{h}^{\varepsilon}\right\|_{p^{\prime}} \leq C_{2}\left(\varepsilon^{p-1}+h^{2 / p^{\prime}}\right) .
$$

The constants $C_{1}, C_{2}$ only depend on $\|\nabla \mathcal{F}(\boldsymbol{D} \boldsymbol{v})\|_{2},\|\pi\|_{1, p^{\prime}}, p, \varepsilon_{0}, \Omega, \boldsymbol{f}$, and $C_{2}$ additionally depends on $\tilde{\beta}(p)$. As a result, for $\varepsilon:=h^{2 / p}$ it follows from (4.14) that

$$
\left\|\boldsymbol{v}-\boldsymbol{v}_{h}^{\varepsilon}\right\|_{1, p} \leq 2 C_{1} h, \quad\left\|\pi-\pi_{h}^{\varepsilon}\right\|_{p^{\prime}} \leq 2 C_{2} h^{2 / p^{\prime}} .
$$

Proof. Since (IS) is fulfilled, the discrete mixed formulation $\left(\mathbf{P}_{h}^{\varepsilon}\right)$ is equivalent to a discrete version of $\left(\mathbf{Q}^{\varepsilon}\right) \equiv\left(\mathbf{M}^{\varepsilon}\right)$. In particular, for each $\varepsilon \geq 0$ the discrete solution $\boldsymbol{v}_{h}^{\varepsilon}$ can be characterized as the unique minimizer of the functional $\mathcal{J}_{\varepsilon}$ in $\mathcal{V}_{h}^{p}$ :

$$
\mathcal{J}_{\varepsilon}\left(\boldsymbol{v}_{h}^{\varepsilon}\right)=\inf _{\boldsymbol{w}_{h} \in \mathcal{V}_{h}^{p}} \mathcal{J}_{\varepsilon}\left(\boldsymbol{w}_{h}\right), \quad \mathcal{V}_{h}^{p}:=\left\{\boldsymbol{w}_{h} \in \mathcal{X}_{h}^{p} ;\left(\operatorname{div} \boldsymbol{w}_{h}, q_{h}\right)_{\Omega}=0 \forall q_{h} \in \mathcal{Q}_{h}^{p}\right\} .
$$

Hence, we can adjust the proof of Theorem 4.1 to the discrete setting. We conclude

$$
\left\|\boldsymbol{D} \boldsymbol{v}_{h}-\boldsymbol{D} \boldsymbol{v}_{h}^{\varepsilon}\right\|_{p} \leq c_{1} \varepsilon^{p / 2}, \quad\left\|\pi_{h}-\pi_{h}^{\varepsilon}\right\|_{p^{\prime}} \leq c_{2} \varepsilon^{p-1},
$$

for some positive constants $c_{1}=c_{1}\left(p, \varepsilon_{0}, \Omega, \boldsymbol{f}\right)$ and $c_{2}=c_{2}(p, \tilde{\beta}(p), \Omega)$. Using the latter inequalities, the error estimates (4.13) with $\varepsilon=0$, and the Korn-Poincaré inequality, we easily deduce the desired estimates (4.14).

Remark 4.4. When we choose finite element pairings $\mathcal{X}_{h}^{p} \times \mathcal{Q}_{h}^{p}$ that do not satisfy the discrete inf-sup condition (IS), then we need to stabilize the Galerkin discretization $\left(\mathbf{P}_{h}^{\varepsilon}\right)$. If standard stabilization methods such as LPS or PSPG are applied (cf. [7]), then the discrete velocity $\boldsymbol{v}_{h}^{\varepsilon}$ can no longer be interpreted as the minimizer of $\mathcal{J}_{\varepsilon}$ in $\mathcal{V}_{h}^{p}$. Hence, we cannot apply Theorem 4.1 to the discrete setting as carried out in Corollary 4.3. However, Theorem 4.1 yields an upper bound of $\left(\boldsymbol{v}-\boldsymbol{v}^{\varepsilon}\right)$ in terms of $\varepsilon$ so that the overall approximation error can be estimated as follows:

$$
\left\|\boldsymbol{v}-\boldsymbol{v}_{h}^{\varepsilon}\right\|_{1, p} \leq\left\|\boldsymbol{v}-\boldsymbol{v}^{\varepsilon}\right\|_{1, p}+\left\|\boldsymbol{v}^{\varepsilon}-\boldsymbol{v}_{h}^{\varepsilon}\right\|_{1, p} \leq c \varepsilon^{\frac{p}{2}}+\left\|\boldsymbol{v}^{\varepsilon}-\boldsymbol{v}_{h}^{\varepsilon}\right\|_{1, p} .
$$

In order to derive an estimate similar to (4.14), the discretization error $\left(\boldsymbol{v}^{\varepsilon}-\boldsymbol{v}_{h}^{\varepsilon}\right)$ needs to be estimated. For this, the error estimate (4.13) is available. Note that the constant $C_{1}$ in (4.13) depends on $\left\|\nabla \mathcal{F}_{\varepsilon}\left(\boldsymbol{D} \boldsymbol{v}^{\varepsilon}\right)\right\|_{2}$ and $\left\|\pi^{\varepsilon}\right\|_{1, p^{\prime}}$. In order to be able to deduce (4.14) 1 from (4.15), we have to assume that there exist constants $C, C^{\prime}>0$ independent of $\varepsilon \in\left[0, \varepsilon_{0}\right]$ such that

$$
\left\|\nabla \mathcal{F}_{\varepsilon}\left(\boldsymbol{D} \boldsymbol{v}^{\varepsilon}\right)\right\|_{2} \leq C, \quad\left\|\nabla \pi^{\varepsilon}\right\|_{p^{\prime}} \leq C^{\prime} .
$$

The question is whether it is allowed to assume (4.16). In fact (4.16) 1 holds true; see Theorem 6.3 in [5. However, assumption (4.16) 2 seems to be rather sophisticated. According to Theorem 6.3 in [5], the pressure gradient $\nabla \pi^{\varepsilon}$ is only bounded in $\boldsymbol{L}^{2}(\Omega)$ by a constant which might explode as $\varepsilon \searrow 0$. Alternatively, in Corollary 4.3 we can avoid assumption (IS) if we employ (4.12) on the discrete level instead of using a discrete version of Theorem 4.1. But then the order of the resulting error estimates is less than the one of (4.14). 


\section{NUMERICAL EXPERIMENTS}

For $p<2, \varepsilon \geq 0$ let the generalized viscosity $\mu$ be given by (1.3) and let the extra stress tensor $\mathcal{S}_{\varepsilon}$ be defined by $\mathcal{S}_{\varepsilon}(\boldsymbol{D} \boldsymbol{v}) \equiv \mu\left(|\boldsymbol{D} \boldsymbol{v}|^{2}\right) \boldsymbol{D} \boldsymbol{v}$. From a numerical point of view, the singular power-law model $(\varepsilon=0)$ is more challenging than its regularized counterpart $(\varepsilon>0)$ : In general, the discrete power-law systems cannot numerically be solved without regularization. In this section, we numerically justify the regularized approximation method proposed by Corollary 4.3 .

Let us consider a planar flow between two steady parallel plates driven by the difference of pressure between inlet and outlet. We assume that $\Omega:=(0, L) \times$ $(-H / 2,+H / 2)$ is a simple channel and that its boundary $\partial \Omega$ consists of a solid part $\Gamma$ (upper and lower edge), of an inflow boundary $S_{1}$ (left edge), and of a free outflow boundary $S_{2}$ (right edge). On the solid part we prescribe homogeneous Dirichlet boundary conditions: $\boldsymbol{v}=\mathbf{0}$ on $\Gamma$. On the inlet and outlet we require

$$
-\frac{\mu\left(|\boldsymbol{D} \boldsymbol{v}|^{2}\right)}{2} \partial_{\boldsymbol{n}} \boldsymbol{v}+\pi \boldsymbol{n}=b_{i} \boldsymbol{n} \quad \text { on } S_{i}, \quad i \in\{1,2\},
$$

for given $b_{i} \in \mathbb{R}$. Here, $\boldsymbol{n}$ denotes the outer normal on $\partial \Omega$, and $\partial_{\boldsymbol{n}} \boldsymbol{v}$ is the corresponding directional derivative. We recall that $\nabla \boldsymbol{v}=\left(\partial_{j} v_{i}\right)_{i, j=1}^{d}$ and $\partial_{\boldsymbol{n}} \boldsymbol{v}=$ $(\boldsymbol{n} \cdot \nabla) \boldsymbol{v}=[\nabla \boldsymbol{v}] \boldsymbol{n}$. The boundary conditions (5.1) arise from the variational formulation and they implicitly normalize the pressure without an additional constraint on its mean value; cf. Remark 5.1. Related to boundary conditions of type (5.1), extensive discussions can be found in [14].

Remark 5.1. Alternatively, we can prescribe the boundary conditions (see [17])

$$
\left.\begin{array}{r}
-\mathcal{S}_{\varepsilon}(\boldsymbol{D} \boldsymbol{v}) \boldsymbol{n} \cdot \boldsymbol{n}+\pi=b_{i} \\
\boldsymbol{v}=(\boldsymbol{v} \cdot \boldsymbol{n}) \boldsymbol{n}
\end{array}\right\} \quad \text { on } S_{i}, \quad i \in\{1,2\} .
$$

In the case of simple channel flows, the boundary conditions (5.1) and (5.2) lead to the same flow behavior: The condition $\boldsymbol{v}=(\boldsymbol{v} \cdot \boldsymbol{n}) \boldsymbol{n}$ on $S_{i}$ ensures that stream lines are orthogonal to the inflow and outflow boundary, i.e., that $\boldsymbol{v}=\left(v_{1}, 0\right)^{\top}$ on $S_{i}$. We note that $\left.\boldsymbol{n}\right|_{S_{i}}$ is a constant vector and that $\boldsymbol{v}=(\boldsymbol{v} \cdot \boldsymbol{n}) \boldsymbol{n}$ implies $\partial_{x_{1}} v_{1}=\operatorname{div} \boldsymbol{v}=0$. Hence, we conclude that $v_{1}=v_{1}\left(x_{2}\right)$ and, consequently, $[\nabla \boldsymbol{v}] \boldsymbol{n}=\mathbf{0}$ on $S_{i}$. Let $v_{n}=(\boldsymbol{v} \cdot \boldsymbol{n})$ be the normal component of $\boldsymbol{v}$. Let $\boldsymbol{t}$ be the tangential vector on $\partial \Omega$, and $v_{t}$ the corresponding tangential component of $\boldsymbol{v}$. Using $[\nabla \boldsymbol{v}] \boldsymbol{n}=\mathbf{0}$ on $S_{i}$ and $[\nabla \boldsymbol{v}]^{\top} \boldsymbol{n}=\nabla v_{n}$, we equivalently write the condition (5.2) as follows:

$$
-\frac{\mu\left(|\boldsymbol{D} \boldsymbol{v}|^{2}\right)}{2} \partial_{\boldsymbol{n}} v_{n}+\pi=b_{i} \quad \text { on } S_{i} .
$$

Integrating this over $S_{i}$, observing $\partial_{\boldsymbol{n}} v_{n}=-\partial_{\boldsymbol{t}} v_{t}(\operatorname{div} \boldsymbol{v}=0)$, we finally arrive at

$$
\int_{S_{i}} \pi \mathrm{d} o=\left|S_{i}\right| b_{i}-\frac{1}{2} \int_{S_{i}} \mu\left(|\boldsymbol{D} \boldsymbol{v}|^{2}\right) \partial_{\boldsymbol{t}} v_{t} \mathrm{~d} o, \quad i \in\{1,2\}
$$

Multiplying (5.1) by $\boldsymbol{n}$ and integrating the result over $S_{i}$, we obtain the condition (5.3) as well. As a result, both (5.1) and (5.2) lead to (5.3). If $\boldsymbol{v}=(\boldsymbol{v} \cdot \boldsymbol{n}) \boldsymbol{n}$ on $S_{i}$, i.e., if $v_{t} \equiv 0$ on $S_{i}$, then $\partial_{t} v_{t}=0$ and, hence, $\int_{S_{i}} \pi \mathrm{d} o=\left|S_{i}\right| b_{i}$. We realize that the prescribed value $b_{i}$ can be interpreted as the mean-value of the pressure over $S_{i}$. 
The weak pressure-drop problem reads as follows: Find a velocity $\boldsymbol{v}^{\varepsilon} \in \mathcal{X}_{\Gamma}^{p}:=$ $\left\{\boldsymbol{w} \in \boldsymbol{W}^{1, p}(\Omega) ;\left.\boldsymbol{w}\right|_{\Gamma}=\mathbf{0}\right\}$ and a pressure $\pi^{\varepsilon} \in \mathcal{Q}_{\Gamma}^{p}:=L^{p^{\prime}}(\Omega)$ satisfying $(\boldsymbol{f} \equiv \mathbf{0})$ :

$$
\begin{aligned}
& \left(\mathcal{S}_{\varepsilon}\left(\boldsymbol{D} \boldsymbol{v}^{\varepsilon}\right), \boldsymbol{D} \boldsymbol{w}\right)_{\Omega}-\left(\pi^{\varepsilon}, \operatorname{div} \boldsymbol{w}\right)_{\Omega}+\left(\operatorname{div} \boldsymbol{v}^{\varepsilon}, q\right)_{\Omega} \\
& =\sum_{i}\left(\frac{\mu\left(\left|\boldsymbol{D} \boldsymbol{v}^{\varepsilon}\right|^{2}\right)}{2}\left[\nabla \boldsymbol{v}^{\varepsilon}\right]^{\top} \boldsymbol{n}-b_{i} \boldsymbol{n}, \boldsymbol{w}\right)_{S_{i}} \quad \forall(\boldsymbol{w}, q) \in \mathcal{X}_{\Gamma}^{p} \times \mathcal{Q}_{\Gamma}^{p} .
\end{aligned}
$$

The weak formulation (5.4) implicitly contains the natural boundary conditions (5.1). Below we set $b_{1}=L / 2$ and $b_{2}=0$. In the case under consideration, it can easily be verified that for $\varepsilon=0$ the unique solution $(\boldsymbol{v}, \pi)$ to (5.4) is given by

$$
v_{1}(\boldsymbol{x})=c_{p}\left(0.5^{\frac{p}{p-1}}-\left|x_{2} / H\right|^{\frac{p}{p-1}}\right), \quad v_{2}(\boldsymbol{x}) \equiv 0, \quad \pi(\boldsymbol{x})=-\frac{1}{2} x_{1}+\frac{1}{2} L
$$

where $c_{p}=\mu_{0}^{-\frac{1}{p-1}} \sqrt{2^{\frac{p-2}{p-1}}} \frac{p-1}{p} H^{\frac{p}{p-1}}$.

The considered pressure-drop problem seems to be a proper example for two reasons: The data such as $\boldsymbol{f}$ are independent of $p$; The function $(\boldsymbol{v}, \pi)$ defined in (5.5) captures the typical flow behavior of a shear thinning fluid: For $1<p \ll 2$ sharp boundary layers occur near $\Gamma$, and the measure of the critical set $\Omega_{c}:=\{\boldsymbol{x} \in$ $\Omega ; \nabla \boldsymbol{v}(\boldsymbol{x}) \approx \mathbf{0}\}$ becomes large. The nonlinear operator associated with $\mathcal{S}_{0}$ is not differentiable on $\Omega_{c}$ so that the convergence of Newton's method is not ensured in general. Hence, numerical problems related to the stability of the solver may be expected when the algebraic equations arising from the FE discretization of (5.4) with $\varepsilon=0$ are solved directly by means of Newton's method. For $1<p \ll 2$ our numerical simulations will indicate that the solution $(\boldsymbol{v}, \pi)$ cannot be numerically approximated via the direct application of the FEM-Newton algorithm but it can be approximated with help of the method proposed by Corollary 4.3 .

Remark 5.2. Below we highlight the structure of the functions that solve the pressure-drop problem under consideration of $\varepsilon \geq 0$. Let $\mu_{0}=1$ and let $\Phi_{\varepsilon}$ be defined in (2.1). We introduce a function $\tilde{\boldsymbol{v}}^{\varepsilon}:(0, L) \times(-H / 2,+H / 2) \rightarrow \mathbb{R}^{2}$ by

$$
\tilde{v}_{1}^{\varepsilon}(\boldsymbol{x}):=\sqrt{2} H\left[\Phi_{\varepsilon}^{*}(0.5)-\Phi_{\varepsilon}^{*}\left(\left|x_{2}\right| / H\right)\right], \quad \Phi_{\varepsilon}^{*}(t):=\int_{0}^{t}\left(\Phi_{\varepsilon}^{\prime}\right)^{-1}(s) \mathrm{d} s, \quad \tilde{v}_{2}^{\varepsilon} \equiv 0 .
$$

If $\varepsilon=0$, then $\Phi_{0}^{*}(t)=\frac{1}{p^{\prime}} t^{p^{\prime}}$ and, hence, $\tilde{\boldsymbol{v}}^{0}$ coincides with $\boldsymbol{v}$ given by (5.5) up to scaling. Note that $\left(\Phi_{\varepsilon}^{*}\right)^{\prime}(t)=\left(\Phi_{\varepsilon}^{\prime}\right)^{-1}(t)$ for $t>0$. Thus, the derivative of $\tilde{v}_{1}^{\varepsilon}$ equals

$$
\left(\tilde{v}_{1}^{\varepsilon}\right)^{\prime}\left(x_{2}\right)=-\sqrt{2} H\left(\Phi_{\varepsilon}^{*}\right)^{\prime}\left(\left|x_{2}\right| / H\right) \frac{x_{2}}{H\left|x_{2}\right|}=-\sqrt{2}\left(\Phi_{\varepsilon}^{\prime}\right)^{-1}\left(\left|x_{2}\right| / H\right) \frac{x_{2}}{\left|x_{2}\right|} .
$$

As a result, the symmetric part of the velocity gradient takes the form

$$
\boldsymbol{D} \tilde{\boldsymbol{v}}^{\varepsilon}(\boldsymbol{x})=\frac{1}{\sqrt{2}}\left(\begin{array}{cc}
0 & -\left(\Phi_{\varepsilon}^{\prime}\right)^{-1}\left(\left|x_{2}\right| / H\right) \frac{x_{2}}{\left|x_{2}\right|} \\
-\left(\Phi_{\varepsilon}^{\prime}\right)^{-1}\left(\left|x_{2}\right| / H\right) \frac{x_{2}}{\left|x_{2}\right|} & 0
\end{array}\right) .
$$

Since $|\boldsymbol{D} \boldsymbol{v}|:=\sqrt{\boldsymbol{D} \boldsymbol{v}: \boldsymbol{D} \boldsymbol{v}}$, we conclude that $\left|\boldsymbol{D} \tilde{\boldsymbol{v}}^{\varepsilon}\right|=\left(\Phi_{\varepsilon}^{\prime}\right)^{-1}\left(\left|x_{2}\right| / H\right)$. Hence,

$$
\mathcal{S}_{\varepsilon}\left(\boldsymbol{D} \tilde{\boldsymbol{v}}^{\varepsilon}\right)=\Phi_{\varepsilon}^{\prime}\left(\left|\boldsymbol{D} \tilde{\boldsymbol{v}}^{\varepsilon}\right|\right) \frac{\boldsymbol{D} \tilde{\boldsymbol{v}}^{\varepsilon}}{\left|\boldsymbol{D} \tilde{\boldsymbol{v}}^{\varepsilon}\right|}=\frac{-\left|x_{2}\right|}{\sqrt{2} H}\left(\begin{array}{cc}
0 & \frac{x_{2}}{\left|x_{2}\right|} \\
\frac{x_{2}}{\left|x_{2}\right|} & 0
\end{array}\right)=\frac{-1}{\sqrt{2} H}\left(\begin{array}{cc}
0 & x_{2} \\
x_{2} & 0
\end{array}\right) .
$$

Clearly, $\operatorname{div} \mathcal{S}_{\varepsilon}\left(\boldsymbol{D} \tilde{\boldsymbol{v}}^{\varepsilon}\right)$ is constant. If $\tilde{\boldsymbol{v}}^{\varepsilon}$ represents the velocity field solving the momentum equations $-\operatorname{div} \mathcal{S}_{\varepsilon}\left(\boldsymbol{D} \tilde{\boldsymbol{v}}^{\varepsilon}\right)+\nabla \tilde{\pi}^{\varepsilon}=\mathbf{0}$, then the pressure field $\tilde{\pi}^{\varepsilon}$ necessarily needs to be a linear function satisfying $\partial_{x_{2}} \tilde{\pi}^{\varepsilon} \equiv 0$. 
TABLE 2. Development of the discretization error $E_{\boldsymbol{v}}^{1, p}$ : Case $\varepsilon=0$

\begin{tabular}{|c|c|c|c|c|c|c|c|c|}
\hline \multirow[b]{2}{*}{ \#cells } & \multicolumn{2}{|c|}{$p=1.1$} & \multicolumn{2}{|c|}{$p=1.2$} & \multicolumn{2}{|c|}{$p=1.3$} & \multicolumn{2}{|c|}{$p=1.5$} \\
\hline & error & conv. & error & conv. & error & conv. & error & conv. \\
\hline $4^{4}$ & $4.55 \mathrm{e}-02$ & - & $6.29 \mathrm{e}-02$ & - & $6.96 \mathrm{e}-02$ & - & $7.48 \mathrm{e}-02$ & - \\
\hline $4^{5}$ & - & - & $3.22 \mathrm{e}-02$ & 0.97 & $3.51 \mathrm{e}-02$ & 0.99 & $3.75 \mathrm{e}-02$ & 1.00 \\
\hline $4^{6}$ & - & - & $1.62 \mathrm{e}-02$ & 0.99 & $1.76 \mathrm{e}-02$ & 1.00 & $1.88 \mathrm{e}-02$ & 1.00 \\
\hline $4^{7}$ & - & - & $8.10 \mathrm{e}-03$ & 1.00 & $8.80 \mathrm{e}-03$ & 1.00 & $9.38 \mathrm{e}-03$ & 1.00 \\
\hline $4^{8}$ & - & - & - & - & $4.40 \mathrm{e}-03$ & 1.00 & $4.69 \mathrm{e}-03$ & 1.00 \\
\hline $4^{9}$ & - & - & - & - & $2.20 \mathrm{e}-03$ & 1.00 & $2.34 \mathrm{e}-03$ & 1.00 \\
\hline expected & & 1.00 & & 1.00 & & 1.00 & & 1.00 \\
\hline
\end{tabular}

Note that the theoretical studies carried out so far only cover the case of homogeneous Dirichlet boundary conditions. However, we believe that they can be generalized to boundary conditions of type (5.2) as long as $|\Gamma|>0$; cf. [16, 17.

Problem (5.4) was discretized with equal-order $\mathbb{Q}_{1} / \mathbb{Q}_{1}$ finite elements based on quadrilateral meshes. Since the considered discretization is not "inf-sup" stable, the following stabilized discrete system was solved:

$$
\begin{aligned}
& \left(\mathcal{S}_{\varepsilon}\left(\boldsymbol{D} \boldsymbol{v}_{h}^{\varepsilon}\right), \boldsymbol{D} \boldsymbol{w}_{h}\right)_{\Omega}-\left(\pi_{h}^{\varepsilon}, \operatorname{div} \boldsymbol{w}_{h}\right)_{\Omega}+s_{h}\left(\pi_{h}^{\varepsilon}, q_{h}\right)+\left(\operatorname{div} \boldsymbol{v}_{h}^{\varepsilon}, q_{h}\right)_{\Omega} \\
& \quad=\sum_{i}\left(\frac{\mu\left(\left|\boldsymbol{D} \boldsymbol{v}_{h}^{\varepsilon}\right|^{2}\right)}{2}\left[\nabla \boldsymbol{v}_{h}^{\varepsilon}\right]^{\top} \boldsymbol{n}-b_{i} \boldsymbol{n}, \boldsymbol{w}_{h}\right)_{S_{i}} \quad \forall\left(\boldsymbol{w}_{h}, q_{h}\right) \in \mathcal{X}_{\Gamma ; h}^{p} \times \mathcal{Q}_{\Gamma ; h}^{p} .
\end{aligned}
$$

Here, the stabilization term $s_{h}\left(\pi_{h}^{\varepsilon}, q_{h}\right)$ was chosen according to the LPS method proposed in 3]. The algebraic equations were solved by Newton's method (see Section 3), the linear subproblems by the GMRES method. A multigrid method with W-cycle was applied as a preconditioner. All computations were performed by means of the software package Gascoigne [13. The parameters were set to $L=1.64, H=0.41, b_{1}=0.82, b_{2}=0, \mu_{0}=0.15$. In our numerical experiments, we measured the approximation errors $E_{\boldsymbol{v}}^{1, p}:=\left\|\nabla\left(\boldsymbol{v}-\boldsymbol{v}_{h}^{\varepsilon}\right)\right\|_{p}, E_{\pi}^{p^{\prime}}:=\left\|\pi-\pi_{h}^{\varepsilon}\right\|_{p^{\prime}}$ and corresponding convergence rates under global mesh refinement.

Example 1. In this example, we did not regularize the singular power-law model and we directly solved the discrete system (5.6) with $\varepsilon=0$ applying Newton's method. Table 2 depicts the discretization errors $\left(\boldsymbol{v}-\boldsymbol{v}_{h}\right)$ with respect to the $\boldsymbol{W}^{1, p}(\Omega)$-norm and corresponding convergence rates for different values of $p$. Note that the pressure $\pi$ belongs to the finite element space $\mathcal{Q}_{\Gamma ; h}^{p}$ and, hence, $\pi$ was resolved exactly up to machine accuracy. Thus convergence rates for the pressure are not presented. For $p \geq 1.3$ we observe that the discretization error behaves as $\mathcal{O}(h)$. This agrees with the theoretical results derived in [4, 15]. For $p<1.3$ we were not able to determine $\boldsymbol{v}_{h}$ numerically using Newton's method. For instance, if $p=1.2$, then the Newton iteration did not reach the prescribed tolerance $T O L=$ $10^{-11}$ for the residual in the case of the mesh with $4^{8}$ elements.

Below we intend to illustrate the approximation method proposed by Corollary 4.3 Instead of solving system (5.6) with $\varepsilon=0$, we determine the solution $\boldsymbol{v}_{h}^{\varepsilon}$ to system (5.6) for small $\varepsilon>0$. For diminishing mesh size $h \searrow 0$, the regularization error, which is caused by regularization of the power-law model with $\varepsilon>0$, dominates the discretization error. In order to obtain a convergent method 
TABLE 3. Development of $E_{\boldsymbol{v}}^{1, p}$ with respect to $\varepsilon_{0}$ : Case $p=1.1$

\begin{tabular}{|c|c|c|c|c|c|c|}
\hline \multirow[b]{2}{*}{ \#cells } & \multicolumn{2}{|c|}{$\varepsilon_{0}=0$} & \multicolumn{2}{|c|}{$\varepsilon_{0}=1$} & \multicolumn{2}{|c|}{$\varepsilon_{0}=10^{2}$} \\
\hline & error & conv. & error & conv. & error & conv. \\
\hline $4^{4}$ & $4.55 \mathrm{e}-02$ & - & $4.64 \mathrm{e}-02$ & - & $6.06 \mathrm{e}-02$ & - \\
\hline $4^{5}$ & - & - & $2.52 \mathrm{e}-02$ & 0.88 & $2.92 \mathrm{e}-02$ & 1.05 \\
\hline $4^{6}$ & - & - & $1.29 \mathrm{e}-02$ & 0.97 & $1.39 \mathrm{e}-02$ & 1.07 \\
\hline $4^{7}$ & - & - & $6.46 \mathrm{e}-03$ & 0.99 & $6.73 \mathrm{e}-03$ & 1.05 \\
\hline $4^{8}$ & - & - & $3.23 \mathrm{e}-03$ & 1.00 & $3.30 \mathrm{e}-03$ & 1.03 \\
\hline $4^{9}$ & - & - & $1.61 \mathrm{e}-03$ & 1.00 & $1.63 \mathrm{e}-03$ & 1.02 \\
\hline expected & & 1.00 & & 1.00 & & 1.00 \\
\hline
\end{tabular}

TABLE 4. Development of $E_{\boldsymbol{v}}^{1, p}$ with respect to $\varepsilon_{0}$ : Case $p=1.2$

\begin{tabular}{|c|c|c|c|c|c|c|}
\hline \multirow[b]{2}{*}{ \#cells } & \multicolumn{2}{|c|}{$\varepsilon_{0}=0$} & \multicolumn{2}{|c|}{$\varepsilon_{0}=1$} & \multicolumn{2}{|c|}{$\varepsilon_{0}=10^{2}$} \\
\hline & error & conv. & error & conv. & error & conv. \\
\hline $4^{4}$ & $6.29 \mathrm{e}-02$ & - & $6.33 \mathrm{e}-02$ & - & $7.61 \mathrm{e}-02$ & - \\
\hline $4^{5}$ & $3.22 \mathrm{e}-02$ & 0.97 & $3.23 \mathrm{e}-02$ & 0.97 & $3.59 \mathrm{e}-02$ & 1.09 \\
\hline $4^{6}$ & $1.62 \mathrm{e}-02$ & 0.99 & $1.62 \mathrm{e}-02$ & 0.99 & $1.71 \mathrm{e}-02$ & 1.07 \\
\hline $4^{7}$ & $8.10 \mathrm{e}-03$ & 1.00 & $8.11 \mathrm{e}-03$ & 1.00 & $8.34 \mathrm{e}-03$ & 1.04 \\
\hline $4^{8}$ & - & - & $4.06 \mathrm{e}-03$ & 1.00 & $4.11 \mathrm{e}-03$ & 1.02 \\
\hline $4^{9}$ & - & - & $2.03 \mathrm{e}-03$ & 1.00 & $2.04 \mathrm{e}-03$ & 1.01 \\
\hline expected & & 1.00 & & 1.00 & & 1.00 \\
\hline
\end{tabular}

for $h \searrow 0$, we couple the parameter $\varepsilon$ with the mesh size $h$ so that we preserve the convergence rate of the discretization error. The choice $\varepsilon=\varepsilon_{0} h^{2 / p}$ implies that the regularization error is of same order as the discretization error at least.

Example 2. We solved the regularized discrete system (5.6) with $\varepsilon=\varepsilon_{0} h^{2 / p}$. It is easy to see that $\pi^{\varepsilon}$ coincides with $\pi$ given by (5.5) for all $\varepsilon \geq 0$. Indeed, $\pi^{\varepsilon}$ is a linear function that satisfies $\partial_{x_{2}} \pi^{\varepsilon}=0$; cf. Remark 5.2. Hence, the condition $\int_{S_{i}} \pi \mathrm{d} o=\left|S_{i}\right| b_{i}$ actually fixes the absolute value of the pressure on the inlet and outlet: $\left.\pi^{\varepsilon}\right|_{S_{i}}=b_{i}$. Consequently, there holds $\pi^{\varepsilon}=\pi$ for all $\varepsilon \geq 0$. Since $\pi$ is linear and hence $\pi \in \mathcal{Q}_{\Gamma: h}^{p}$, the pressure was resolved exactly up to machine accuracy: $\pi_{h}^{\varepsilon}=\pi$. Tables 3 and 4 depict the approximation errors $\left(\boldsymbol{v}-\boldsymbol{v}_{h}^{\varepsilon}\right)$ in $\boldsymbol{W}^{1, p}(\Omega)$ with $\varepsilon=\varepsilon_{0} h^{2 / p}$. Since the pressure was resolved exactly, only velocity errors are presented. Independently of the value of $\varepsilon_{0}$, we expect that $E_{\boldsymbol{v}}^{1, p}$ behaves as $\mathcal{O}(h)$ due to Corollary 4.3. Considering Tables 3 and 4, we realize that the numerical results agree with the theoretical ones very well. In the case of $\varepsilon_{0}=0$, the numerical results coincide with those from Example 1. The missing numbers indicate that Newton's method did not converge. More precisely, the Newton iteration did not reach the prescribed tolerance $T O L=10^{-11}$ for the residual. Comparing the absolute errors for $\varepsilon_{0}=0$ with those for $\varepsilon_{0} \neq 0$ depicted in Tables 3 and 4 we finally observe that, despite additional regularization errors, the proposed approximation method leads to higher accuracy compared to the nonregularized FEM. 
Example 3. We considered another flow configuration which is less realistic from a physical point of view but which exhibits a nonsmooth analytical solution. Here, we chose the computational domain $\Omega:=(-0.5,0.5)^{2}$ and we defined $\boldsymbol{v}(\boldsymbol{x}):=$ $|\boldsymbol{x}|^{7}\left(x_{1},-x_{2}\right)^{\top}$ and $\pi(\boldsymbol{x}):=|\boldsymbol{x}|^{b} x_{1} x_{2}$. The right-hand side $\boldsymbol{f}$ was chosen accordingly as $\boldsymbol{f}:=-\operatorname{div} \mathcal{S}(\boldsymbol{D} \boldsymbol{v})+\nabla \pi$, and system (1.1) was complemented with nonhomogeneous Dirichlet boundary conditions: On $\partial \Omega$ the boundary values $\left.\boldsymbol{v}\right|_{\partial \Omega}$ were prescribed. The parameter $b$ was chosen so that $\pi \in W^{1, p^{\prime}}(\Omega)$. This condition is ensured for $b>-\frac{2}{p^{\prime}}-1$. We approximatively solved the corresponding weak boundary value problem for the following parameters: $p=1.3, \mu_{0}=1$, and $b=-1.45$. Table 5 depicts the approximation errors $\left(\boldsymbol{v}-\boldsymbol{v}_{h}^{\varepsilon}\right)$ and corresponding convergence rates for $\varepsilon=\varepsilon_{0} h^{2 / p}$. We realize that $\boldsymbol{v}_{h}^{\varepsilon}$ converges to $\boldsymbol{v}$ in $\boldsymbol{W}^{1, p}(\Omega)$ with at least order one. The numerical results for the pressure are presented in Table 6. We observe that the error $\left(\pi-\pi_{h}^{\varepsilon}\right)$ measured in $L^{p^{\prime}}(\Omega)$ behaves as $\mathcal{O}(h)$. In view of Tables 5 and 6, the numerical results agree with the theoretical ones stated in Corollary 4.3 .

TABle 5. Development of $E_{\boldsymbol{v}}^{1, p}$ with respect to $\varepsilon_{0}$ : Case $p=1.3$

\begin{tabular}{|c|c|c|c|c|c|c|}
\hline \multirow[b]{2}{*}{ \#cells } & \multicolumn{2}{|c|}{$\varepsilon_{0}=0$} & \multicolumn{2}{|c|}{$\varepsilon_{0}=1$} & \multicolumn{2}{|c|}{$\varepsilon_{0}=20$} \\
\hline & error & conv. & error & conv. & error & conv. \\
\hline $4^{4}$ & $1.12 \mathrm{e}-02$ & - & $1.47 \mathrm{e}-02$ & - & $3.52 \mathrm{e}-02$ & - \\
\hline $4^{5}$ & $5.54 \mathrm{e}-03$ & 1.02 & $6.47 \mathrm{e}-03$ & 1.18 & $1.37 \mathrm{e}-02$ & 1.37 \\
\hline $4^{6}$ & $2.76 \mathrm{e}-03$ & 1.01 & $2.99 \mathrm{e}-03$ & 1.11 & $5.05 \mathrm{e}-03$ & 1.44 \\
\hline $4^{7}$ & $1.37 \mathrm{e}-03$ & 1.00 & $1.43 \mathrm{e}-03$ & 1.06 & $1.95 \mathrm{e}-03$ & 1.37 \\
\hline $4^{8}$ & $6.87 \mathrm{e}-04$ & 1.00 & $7.00 \mathrm{e}-04$ & 1.03 & $8.27 \mathrm{e}-04$ & 1.24 \\
\hline $4^{9}$ & $3.43 \mathrm{e}-04$ & 1.00 & $3.46 \mathrm{e}-04$ & 1.02 & $3.76 \mathrm{e}-04$ & 1.14 \\
\hline sipecter & & 1.00 & & 1.00 & & 1.00 \\
\hline
\end{tabular}

TABLE 6. Development of $E_{\pi}^{p^{\prime}}$ with respect to $\varepsilon_{0}$ : Case $p=1.3$

\begin{tabular}{|c|c|c|c|c|c|c|}
\hline \multirow[b]{2}{*}{ \#cells } & \multicolumn{2}{|c|}{$\varepsilon_{0}=0$} & \multicolumn{2}{|c|}{$\varepsilon_{0}=1$} & \multicolumn{2}{|c|}{$\varepsilon_{0}=20$} \\
\hline & error & conv. & error & conv. & error & conv. \\
\hline $4^{4}$ & $2.84 \mathrm{e}-02$ & - & $2.66 \mathrm{e}-02$ & - & $3.63 \mathrm{e}-02$ & - \\
\hline $4^{5}$ & $1.28 \mathrm{e}-02$ & 1.45 & $1.14 \mathrm{e}-02$ & 1.22 & $1.17 \mathrm{e}-02$ & 1.63 \\
\hline $4^{6}$ & $6.37 \mathrm{e}-03$ & 1.01 & $5.47 \mathrm{e}-03$ & 1.06 & $5.19 \mathrm{e}-03$ & 1.17 \\
\hline $4^{7}$ & $3.22 \mathrm{e}-03$ & 0.98 & $2.67 \mathrm{e}-03$ & 1.03 & $2.53 \mathrm{e}-03$ & 1.04 \\
\hline $4^{8}$ & $1.60 \mathrm{e}-03$ & 1.01 & $1.31 \mathrm{e}-03$ & 1.02 & $1.24 \mathrm{e}-03$ & 1.03 \\
\hline $4^{9}$ & $7.96 \mathrm{e}-04$ & 1.01 & $6.46 \mathrm{e}-04$ & 1.02 & $6.08 \mathrm{e}-04$ & 1.03 \\
\hline expected & & 0.46 & & 0.46 & & 0.46 \\
\hline
\end{tabular}

Numerical complexity. Finally, for Examples 1-3 we compare the proposed regularized approximation method with the nonregularized one regarding numerical complexity. The numerical costs were measured by the number of iterations carried out by Newton's algorithm. In Table 7 we depict the number of Newton iterations that were performed in order to reduce the residual up to the prescribed tolerance $T O L=10^{-11}$ for each refinement level $L$. Here, $L=1$ corresponds to the mesh 
with $4^{4}$ cells. The number within the brackets represents the total number of iterations performed by the step-size control and it equals the number $l^{*}$ that appears in the Newton algorithm stated in Section 3 for $\lambda=3 / 4$. As an initial guess for Newton's method on level $L$, we chose the FE solution corresponding to level $L-1$. In particular, as initial guess for Newton's method on level $L=1$, we took the discrete solution on the mesh with $4^{3}$ cells. Comparing the number of iterations for $\varepsilon_{0}=0$ with those for $\varepsilon_{0} \neq 0$ depicted in Table 7 we observe that the solution of the nonregularized systems $\left(\varepsilon_{0}=0\right)$ requires more iterations of the Newton algorithm and step-size control than the solution of the regularized systems $\left(\varepsilon_{0} \neq 0\right)$. We recall that in view of Tables 3 and 4 we achieved higher accuracy for reasonable values of $\varepsilon_{0}>0$. Hence, we realize that the approximation method proposed by Corollary 4.3 is more efficient than the standard (nonregularized) FEM.

TABLE 7. Total number of Newton iterations $\left(T O L=10^{-11}\right)$ with respect to the refinement level $L$

(a) Example with $p=1.1$

\begin{tabular}{|c|c|c|c|c|c|c|c|}
\hline$L$ & $\varepsilon_{0}=0$ & $\varepsilon_{0}=1$ & $\varepsilon_{0}=10^{2}$ & $L$ & $\varepsilon_{0}=0$ & $\varepsilon_{0}=1$ & $\varepsilon_{0}=10^{2}$ \\
\hline 1 & $8(11)$ & $6(2)$ & $5(3)$ & 1 & $6(4)$ & $5(2)$ & $5(1)$ \\
\hline 2 & - & $5(2)$ & $6(2)$ & 2 & $6(4)$ & $5(1)$ & $5(1)$ \\
\hline 3 & - & $5(2)$ & $5(2)$ & 3 & $6(4)$ & $5(1)$ & $5(1)$ \\
\hline 4 & - & $5(2)$ & $5(2)$ & 4 & $7(4)$ & $5(1)$ & $5(1)$ \\
\hline 5 & - & $5(2)$ & $5(2)$ & 5 & - & $5(1)$ & $5(1)$ \\
\hline 6 & - & $6(2)$ & $5(2)$ & 6 & - & $4(1)$ & $4(1)$ \\
\hline
\end{tabular}

(c) Example with $p=1.3$

\begin{tabular}{cccc}
\hline$\frac{\varepsilon_{0}=0}{1}$ & $\frac{\varepsilon_{0}=1}{12(6)}$ & $\frac{\varepsilon_{0}=20}{7(0)}$ & $\frac{6(0)}{6(0)}$ \\
2 & $17(11)$ & $5(0)$ & $7(0)$ \\
3 & $16(13)$ & $5(0)$ & $6(0)$ \\
4 & $19(16)$ & $5(0)$ & $5(0)$ \\
5 & $21(18)$ & $5(0)$ & $5(0)$ \\
6 & $16(17)$ & $5(0)$ & $5(0)$ \\
\hline
\end{tabular}

Conclusion. In this paper we studied singular power-law systems and their numerical approximation. The application of Newton's method usually suffers from instabilities. We proposed a numerical method that is based on a simple regularization of the singular power-law model and which enables the stable approximation of singular power-law systems via Newton's method. In Corollary 4.3 we derived a priori error estimates that quantify the convergence of the proposed method. We practically validated them by numerical experiments. The numerical examples indicate that our regularized approximation method surpasses the nonregularized one regarding accuracy and numerical efficiency. 


\section{REFERENCES}

1. C. Amrouche and V. Girault, Decomposition of vector-spaces and application to the Stokes problem in arbitrary dimension, Czechoslovak Math. J. 44 (1994), no. 1, 109-140. MR.1257940 (95c:35190)

2. J. W. Barrett and W. B. Liu, Quasi-norm error bounds for the finite element approximation of a non-Newtonian flow, Numer. Math. 68 (1994), 437-456. MR.1301740 (95h:65078)

3. R. Becker and M. Braack, A finite element pressure gradient stabilization for the Stokes equations based on local projections, Calcolo 38 (2001), 173-199. MR.1890352 (2002m:65112)

4. L. Belenki, L. C. Berselli, L. Diening and M. Růžička, On the finite element approximation of p-Stokes systems, SIAM J. Numer. Anal. 50 (2012), no. 2, 373-397. MR2914267

5. L. C. Berselli, L. Diening and M. Růžička, Existence of strong solutions for incompressible fluids with shear dependent viscosities, J. Math. Fluid Mech. 12 (2010), 101-132. MR2602916 (2011c:76005)

6. D. V. Boger, A. Cabelli and A. L. Halmos, The behavior of a power-law fluid flowing through a sudden expansion, AIChE Journal 21 (1975), no. 3, 540-549.

7. M. Braack, E. Burman, V. John and G. Lube, Stabilized finite element methods for the generalized Oseen problem, Comput. Methods Appl. Mech. Engrg. 196 (2007), 853-866. MR2278180 (2007i:76065)

8. S. Brenner and R. L. Scott, The Mathematical Theory of Finite Element Methods, SpringerVerlag, New York, 1994. MR1278258 (95f:65001)

9. P. G. Ciarlet, The finite elements methods for elliptic problems, North-Holland, 1980.

10. P. Deuflhard, Newton-methods for nonlinear problems - affine invariance and adaptive algorithms, Springer-Verlag, Berlin Heidelberg, 2004. MR2063044 (2005h:65002)

11. L. Diening and F. Ettwein, Fractional estimates for non-differentiable elliptic systems with general growth, Forum Math. 20 (2008), 523-556. MR.2418205(2009h:35101)

12. G. P. Galdi, R. Rannacher, A. M. Robertson and S. Turek, Hemodynamical Flows - Modeling, Analysis and Simulation, Oberwolfach Seminars, vol. 37, Birkhäuser, Basel, 2008. MR2416195 (2009e:76003)

13. GASCOIGNE, The finite element toolkit, http://www.gascoigne.uni-hd.de.

14. J. G. Heywood, R. Rannacher and S. Turek, Artificial boundaries and flux and pressure conditions for the incompressible Navier-Stokes equations, Int. J. Num. Meth. Fluids 22 (1996), 325-352. MR1380844 (97f:76045)

15. A. Hirn, Approximation of the p-Stokes equations with equal-order finite elements, J. Math. Fluid Mech. (2012), doi:10.1007/s00021-012-0095-0.

16. A. Hirn, M. Lanzendörfer and J. Stebel, Finite element approximation of flow of fluids with shear rate and pressure dependent viscosity, IMA Journal of Numerical Analysis (2012), doi: 10.1093/imanum/drr033.

17. M. Lanzendörfer and J. Stebel, On pressure boundary conditions for steady flows of incompressible fluids with pressure and shear rate dependent viscosities, Appl. Math. 56 (2011), no. 3, 265-285. MR 2800578

18. J. Málek, J. Nečas, M. Rokyta, and M. Růžička, Weak and measure-valued solutions to evolutionary PDEs, Chapman \& Hall, London, 1996. MR1409366 (97g:35002)

19. J. Málek and K. R. Rajagopal, Handbook of Differential Equations: Evolutionary equations, vol. 2, Chapter 5, Mathematical issues concerning the Navier-Stokes equations and some of its generalizations, pp. 371-459, Elsevier/North-Holland, Amsterdam, 2005. MR 2182831 (2006k:35221)

20. J. Málek, K. R. Rajagopal and M. Růžička, Existence and regularity of solutions and the stability of the rest state for fluids with shear dependent viscosity, Math. Models Methods Appl. Sci. 5 (1995), 789-812. MR1348587 (96i:76002)

21. L. J. Sonder and P. C. England, Vertical averages of rheology of the continental lithosphere, Earth Planet. Sci. Lett. 77 (1986), 81-90.

Institut für Angewandte Mathematik, Ruprecht-Karls-Universität Heidelberg, Im

Neuenheimer Feld 294, 69120 Heidelberg, Germany

E-mail address: adrian.hirn@iwr.uni-heidelberg.de 